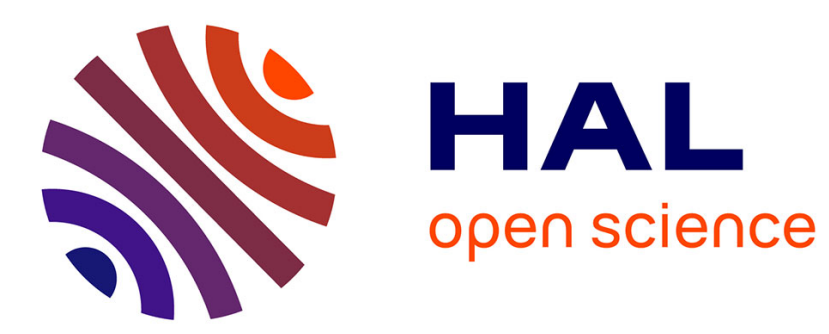

\title{
The suspension balance model revisited
}

Prabhu R Nott, Elisabeth Guazzelli, Olivier Pouliquen

\section{To cite this version:}

Prabhu R Nott, Elisabeth Guazzelli, Olivier Pouliquen. The suspension balance model revisited. Physics of Fluids, 2011, 23, pp.43304 - 43304. 10.1063/1.3570921 . hal-01432494

\section{HAL Id: hal-01432494 https://hal.science/hal-01432494}

Submitted on 11 Jan 2017

HAL is a multi-disciplinary open access archive for the deposit and dissemination of scientific research documents, whether they are published or not. The documents may come from teaching and research institutions in France or abroad, or from public or private research centers.
L'archive ouverte pluridisciplinaire $\mathbf{H A L}$, est destinée au dépôt et à la diffusion de documents scientifiques de niveau recherche, publiés ou non, émanant des établissements d'enseignement et de recherche français ou étrangers, des laboratoires publics ou privés. 


\title{
The suspension balance model revisited
}

\author{
Prabhu R. Nott, ${ }^{1}$ Elisabeth Guazzelli, ${ }^{2}$ and Olivier Pouliquen ${ }^{2}$ \\ ${ }^{1}$ Department of Chemical Engineering, Indian Institute of Science, Bangalore 560 012, India \\ ${ }^{2}$ Institut Universitaire des Systèmes Thermiques Industriels (UMR 6595), Centre National de la Recherche \\ Scientifique, Polytech'Marseille, Aix-Marseille Université, 13453 Marseille cedex 13, France
}

(Received 1 May 2010; accepted 8 March 2011; published online 26 April 2011)

\begin{abstract}
This paper addresses a fundamental discrepancy between the suspension balance model and other two-phase flow formulations. The former was proposed to capture the shear-induced migration of particles in Stokesian suspensions, and hinges on the presence of a particle phase stress to drive particle migration. This stress is taken to be the "particle stress," defined as the particle contribution to the suspension stress. On the other hand, the two-phase flow equations derived in several studies show only a force acting on the particle phase, but no stress. We show that the identification of the particle phase stress with the particle contribution to the suspension stress in the suspension balance model is incorrect, but there exists a well-defined particle phase stress. Following the rigorous method of volume averaging, we show that the force on the particle phase may be written as the sum of an interphase drag and the divergence of the particle phase stress. We derive exact micromechanical relations for these quantities. We also comment on the interpretations and results of previous studies that are based on the identification of the particle phase stress with the particle contribution to the suspension stress. (C) 2011 American Institute of Physics.
\end{abstract}

[doi:10.1063/1.3570921]

\section{INTRODUCTION}

Over the past three decades, several attempts have been made to explain the phenomenon of shear-induced migration of particles in Stokesian suspensions using continuum mechanical models. The term "Stokesian suspension" refers to a suspension for which $\operatorname{Re}_{\mathrm{p}} \equiv \rho u_{0} a / \eta \ll 1$ and $\mathrm{Pe}$ $\equiv 6 \pi \eta a^{2} u_{0} /(k T) \gg 1$, where $\operatorname{Re}_{\mathrm{p}}$ is the Reynolds number based on the particle size $a$, and Pe is the Péclet number. Here, $\rho$ and $\eta$ are the density and viscosity, respectively, of the fluid, $u_{0}$ is a characteristic velocity of the particles, $k$ is the Boltzmann constant, and $T$ is the temperature in the absolute scale. Of the proposed theories, the most frequently used and quoted in the literature are the diffusive flux model proposed by Leighton and Acrivos, ${ }^{1}$ and the suspension balance model proposed by Nott and Brady. ${ }^{2}$ Both models supplement the equations of motion for the suspension with equations that describe the motion of the particles. The diffusive flux model is purely kinematic, in the sense that the flux of the particles in the suspension is described in terms of the gradients of the particle concentration and the strain rate. The suspension balance model attempts to relate the rheology of the suspension with the migration flux of the particles. Nott and Brady ${ }^{2}$ showed that the diffusive flux model can be recovered from the suspension balance model, thereby showing that the two models share the same physical origin. This paper addresses the suspension balance model (hereafter referred to as the SBM), but insofar as the two models are connected, its conclusions do have a bearing on the diffusive flux model.

The SBM captures shear-induced migration by treating the particles as a separate phase: the equations that determine the flux of particles with respect to the suspension are the volume-averaged balances of mass and momentum for the particle phase. It is argued that the shear-induced drift velocity of the particles (i.e., velocity orthogonal to the suspension velocity) is driven by the divergence of the particle phase stress $\langle\boldsymbol{\sigma}\rangle^{\mathrm{p}}$. Thus, the particle phase stress is a crucial element of the SBM. Nott and Brady ${ }^{2}$ equated the particle phase stress to the "particle stress" $\Sigma^{(\mathrm{p})}$, which was introduced by Batchelor ${ }^{3}$ to denote the particle contribution to the suspension stress. For a uniformly sheared Stokesian suspension Batchelor $^{3}$ showed that the hydrodynamic part of the $\mathbf{\Sigma}^{(\mathrm{p})}$ equals $n\left\langle\overline{\boldsymbol{S}}^{\mathrm{h}}\right\rangle$, where $n$ is the number density of particles, $\overline{\boldsymbol{S}}^{\mathrm{h}}$ is the stresslet (a moniker he introduced for the symmetric part of the first moment of the fluid traction on the particle surface), and the angle brackets denote an average over all particles.

On the other hand, equations of motion for general twophase flows have been derived by many workers ${ }^{4-8}$. Jackson ${ }^{6}$ gives a lucid account of the volume averaging approach, based on the earlier work of Anderson and Jackson, ${ }^{9}$ and accounts of the ensemble averaging approach may be found in Buyevich and Shchelchkova ${ }^{4}$ and Zhang and Prosperetti. ${ }^{7}$ Not surprisingly, both approaches yield the same forms of the equations of motion; their result for Stokesian suspensions that is most relevant to the present paper is that the effect of the fluid is only felt as an average hydrodynamic force on the particles-there is no hydrodynamic contribution to the particle phase stress. This force has traditionally been assumed to be the interphase drag, proportional to the difference in velocity between the two phases. Thus, the equation of the hydrodynamic parts of $\langle\boldsymbol{\sigma}\rangle^{\mathrm{p}}$ and $\mathbf{\Sigma}^{(\mathrm{p})}$ in the SBM is incorrect. This seemingly poses a dilemma: if there is no particle phase stress, how can shear-induced migration be explained?

The above discrepancy between the suspension balance 
model and the other two-phase flow approaches was recently pointed out by Lhuillier. ${ }^{10}$ To resolve the above dilemma, he proposed that the average force on the particles is not simply the interphase drag, but has an additional part that drives shear-induced and "stress-induced" migration. Lhuillier ${ }^{10}$ gave a phenomenological expression for the "extra force," and identified a part of it as being the Faxen contribution to the force on a particle that arises from gradients in the velocity field.

In this paper, we reaffirm the statement of Lhuillier ${ }^{10}$ that the hydrodynamic part of $\mathbf{\Sigma}^{(p)}$ (henceforth represented as $\Sigma^{h(p)}$, for brevity) does not find a place in the particle phase momentum balance, and therefore cannot be responsible for driving particle migration. However, we show that there is a well-defined hydrodynamic contribution to the particle phase stress, and argue that it is this that drives particle migration. By following the rigorous volume averaging approach, ${ }^{6,9}$ we show that the hydrodynamic force on the particle phase $n\left\langle f^{\mathrm{h}}\right\rangle^{\mathrm{p}}$ may be written as the sum of a force and the divergence of a stress. We identify the former as the interphase drag $n\left\langle f^{\mathrm{h}}\right\rangle_{\text {drag }}$, and the latter as the hydrodynamic part of the particle phase stress $n\left\langle\boldsymbol{\sigma}^{\mathrm{h}}\right\rangle^{\mathrm{p}}$. This partitioning of $\left\langle f^{\mathrm{h}}\right\rangle^{\mathrm{p}}$ is unique, and is analogous to the derivation of the stress due to nonhydrodynamic forces, such as the forces due to contact, electrostatics etc. Needless to say, the total particle phase stress $n\langle\boldsymbol{\sigma}\rangle^{\mathrm{p}}$ is the sum of the hydrodynamic and nonhydrodynamic parts. Thus, though the identification of $n\left\langle\boldsymbol{\sigma}^{\mathrm{h}}\right\rangle \mathrm{p}$ with $\boldsymbol{\Sigma}^{\mathrm{h}(\mathrm{p})}$ is incorrect, the form of the equations of motion in the SBM is correct. This perhaps explains the success of the SBM in explaining shear-induced particle migration in several flows. We provide exact micromechanical expressions for the interphase drag and the particle phase stress. We show that $\left\langle\boldsymbol{\sigma}^{\mathrm{h}}\right\rangle^{\mathrm{p}}$ is caused solely by hydrodynamic interactions, in conformity with the widely accepted view that particle migration is a result of many-body hydrodynamic interactions. As an aside, we note that most two-phase flow theories use closure models wherein the volume-averaged stress in each phase depends on the volume-averaged kinematic variables of that phase alone-our expressions for the volumeaveraged stresses show that such an assumption is not generally valid.

Several previous studies ${ }^{11-16}$ have attempted to determine parts of the particle phase stress by experiment, simulation, or analysis, by equating $\left\langle\boldsymbol{\sigma}^{\mathrm{h}}\right\rangle^{\mathrm{p}}$ with $\boldsymbol{\Sigma}^{\mathrm{h}(\mathrm{p})}$. In view of our result, their results and interpretations are at least partly in error. Jeffrey et al. ${ }^{11}$ determined the isotropic part of the stresslet $\overline{\boldsymbol{S}}^{\mathrm{h}}$ on a pair of particles, with the intention of calculating the particle phase pressure $\langle p\rangle^{\mathrm{p}}$ using the relation

$$
\langle p\rangle^{\mathrm{p}}=-\operatorname{Tr}\left(\boldsymbol{\Sigma}^{\mathrm{h}(\mathrm{p})}\right) .
$$

The right-hand side of Eq. (1) is a well-defined quantity, and has been interpreted as the non-equilibrium osmotic pressure of the suspension; ${ }^{17}$ however, it is not the particle phase pressure, as this paper demonstrates. Singh and Nott ${ }^{13}$ and Sierou and Brady ${ }^{15}$ attempted to determine $\langle p\rangle^{\mathrm{p}}$ in simple shear flow by Stokesian dynamics simulations, the former by determining the force on the confining walls, and the latter using Eq. (1) — what they actually measured is a part of the suspension pressure. Indeed, Singh and Nott ${ }^{16}$ realized that they could not determine the particle phase pressure from rheological measurements of the suspension. The normal stress differences measured by simulation ${ }^{13,15}$ and experiment ${ }^{14,16}$ are useful viscometric properties of the suspension. Nott and Brady $^{2}$ saw the possibility of normal stress differences of the particle phase modulating particle migration in curvilinear flows, which was shown more clearly by Morris and Boulay ${ }^{18}$ using proposed functional forms for the normal stress differences. However, the normal stress differences of the particle phase are not the same as that of the suspension. The numerous studies ${ }^{19-27}$ that have used and extended the SBM have retained the error of equating $\left\langle\boldsymbol{\sigma}^{\mathrm{h}}\right\rangle^{\mathrm{p}}$ with $\boldsymbol{\Sigma}^{\mathrm{h}(\mathrm{p})}$.

The outline of this paper is as follows. In §II, we write down the equations of motion of the suspension and the particle phase. The volume averaging approach used to derive these equations has been described at length in earlier studies, ${ }^{5,6,9}$ but we repeat it here for the sake of completeness. We largely follow the approach of Jackson, ${ }^{6}$ but deviate in some ways to make contact with the studies of Batchelor ${ }^{3}$ and Nott and Brady. ${ }^{2}$ Neglecting inertial effects, we arrive at momentum balances for the solid and fluid phases, and for the entire suspension-the complete balances, with the effects of particle and fluid inertia included, are given in Appendix A. In the momentum balance for the solid phase, we find that a part of the volume-averaged stress $\phi\langle\boldsymbol{\sigma}\rangle^{\mathrm{s}}$ cancels with a part of the traction at the particle-fluid interfaces what remains is the particle-averaged net force on the particles $\langle f\rangle^{\mathrm{p}}$. The force $\langle f\rangle^{\mathrm{p}}$ has parts due to the hydrodynamic and contact traction on the surface of the particles, which we call $\left\langle f^{\mathrm{h}}\right\rangle^{\mathrm{p}}$ and $\left\langle f^{\mathrm{c}}\right\rangle^{\mathrm{p}}$, respectively. By suitable simplification, we show that the solid phase momentum balance reduces to the particle phase momentum balance, defined first by Anderson and Jackson. ${ }^{9}$ The main advance of this paper is in §III, where we show that the hydrodynamic force on the particle phase $\left\langle f^{\mathrm{h}}\right\rangle^{\mathrm{p}}$ may be written as the sum of a interphase drag force $\left\langle f^{\mathrm{h}}\right\rangle_{\text {drag }}$, and the divergence of a particle phase stress $\left\langle\boldsymbol{\sigma}^{\mathrm{h}}\right\rangle^{\mathrm{p}}$. We derive exact micromechanical relations for $\left\langle\boldsymbol{f}^{\mathrm{h}}\right\rangle_{\text {drag }}$ and $\left\langle\boldsymbol{\sigma}^{\mathrm{h}}\right\rangle^{\mathrm{p}}$, neither of which involve the stresslet; indeed, we show that the stresslet contributes only to the stresses of the fluid phase and the suspension. Thus, the hydrodynamic parts of the particle phase stress $\langle\boldsymbol{\sigma}\rangle^{\mathrm{p}}$ and the "particle stress" $\boldsymbol{\Sigma}^{(\mathrm{p})}$ are not directly related. We also comment on the Brownian contribution to the particle and fluid phase stresses. We note that though our main interest in this paper is on Stokesian suspensions, our procedure for the determination of the volume-averaged solid/particle and fluid phases holds for all suspensions of rigid particles in a Newtonian fluid. We end by summarizing the main results of this paper in $\S \mathrm{V}$, where we also give plausible reasons for the success of the suspension balance model in predicting particle migration (despite the aforementioned error), and comment on ways to extract closure relations for the particle phase stress.

\section{VOLUME-AVERAGED EQUATIONS OF MOTION}

We consider a suspension of rigid spherical particles of radius $a$ in a Newtonian fluid, and take the densities $\rho^{\mathrm{p}}$ and $\rho^{\mathrm{f}}$ 
of the particles and fluid, respectively, to be constants. The particles and fluid occupy the volumes $V^{\mathrm{p}}$ and $V^{\mathrm{f}}$, respectively, the boundaries of which change with time due to the motion of the particles. As in earlier studies, ${ }^{5}$ we define the phase indicator function $\chi$

$$
\chi(y)= \begin{cases}1 & \text { if } \boldsymbol{y} \in V^{\mathrm{p}} \\ 0 & \text { if } \boldsymbol{y} \in V^{\mathrm{f}}\end{cases}
$$

where $y$ is the vector of spatial coordinates in the laboratory reference frame. Assuming no exchange of mass between the fluid and particles, $\chi$ has the properties ${ }^{5}$

$$
\begin{aligned}
& \frac{\mathrm{D} \chi}{\mathrm{D} t}=0, \\
& \boldsymbol{\nabla}_{y} \chi=-\boldsymbol{n} \delta\left(\boldsymbol{y}-\boldsymbol{y}_{s}\right),
\end{aligned}
$$

where $\delta(y)$ is the Dirac delta function, $\boldsymbol{y}_{S}$ are points on the surface of the particles, $\boldsymbol{n}$ the unit outward normal at $\boldsymbol{y}_{s}$, and $\mathrm{D} / \mathrm{D} t \equiv \partial / \partial t+\boldsymbol{u} \cdot \nabla_{y}$ is the material derivative.

To define volume-averaged properties and develop the equations governing them, we follow Anderson and Jackson ${ }^{9}$ in introducing a smoothing (or weighting) function $g(\boldsymbol{x}, \boldsymbol{y})$ which has the property

$$
\int g(\boldsymbol{x}, \boldsymbol{y}) \mathrm{d} V=1
$$

at every point $\boldsymbol{x}$, where the integration is over the entire volume $V \equiv V^{\mathrm{f}}+V^{\mathrm{p}}$ over the space $\boldsymbol{y}$. Here and hereafter, we denote by $\boldsymbol{y}$ the spatial domain of the original (unaveraged) variables, and by $\boldsymbol{x}$ the spatial domain of the averaged variables.

We choose a smoothing function whose form depends only on $\boldsymbol{r} \equiv \boldsymbol{y}-\boldsymbol{x}$, the simplest of which is the isotropic form $g(|\boldsymbol{r}|)$. Consequently, we have the relation ${ }^{6,9}$

$$
\nabla_{x} g=-\nabla_{y} g,
$$

where the subscripts indicate the variable with respect to which the gradient is taken; this identity is of considerable utility while deriving the volume-averaged equations of motion. For ease of notation, we shall henceforth represent $\boldsymbol{\nabla}_{y}$ as $\hat{\boldsymbol{\nabla}}$, and $\boldsymbol{\nabla}_{x}$ as $\boldsymbol{\nabla}$. Finally, we require $g$ and all its gradients to be well-defined, and

$$
g \rightarrow 0 \text { as }|\boldsymbol{r}| \rightarrow \infty
$$

so that the volume average of a property at $\boldsymbol{x}$ is a reflection of its values only in the vicinity of $\boldsymbol{x}$. This statement can be formalized by defining the radius $\ell$ of $g$ as, ${ }^{6,9}$

$$
1 / 2=\int_{|r|<\ell} g(\boldsymbol{x}, \boldsymbol{y}) \mathrm{d} V .
$$

The validity of the volume-averaged equations hinges on the separation of scales

$$
a \ll \ell \ll L
$$

where $L$ is the length scale of macroscopic spatial gradients. When such a separation of scales exists, the volumeaveraged properties do not depend strongly on the exact form of the smoothing function. ${ }^{6,9}$ For some particular cases, such as time-independent flows, the condition $a \ll \ell$ may be relaxed by averaging over a sufficiently long period of time; this procedure is adopted routinely in particle dynamics simulations, where computational constraints place restrictions on the system size.

The volume average $\langle\xi\rangle$ of a microscopically varying property $\xi(\boldsymbol{y})$ over the entire suspension (particles and fluid) at a point $\boldsymbol{x}$ is defined as

$$
\langle\xi\rangle(\boldsymbol{x})=\int \xi(\boldsymbol{y}) g(\boldsymbol{x}, \boldsymbol{y}) \mathrm{d} V .
$$

From (8), it is clear that $\langle\xi\rangle$ reflects the average of $\xi$ in a sphere of radius $\sim \ell$ centered at $\boldsymbol{x}$. It then follows that the particle volume fraction $\phi$ is given by

$$
\phi(x)=\int \chi(\boldsymbol{y}) g(\boldsymbol{x}, \boldsymbol{y}) \mathrm{d} V .
$$

Volume averages over the particles and the fluid are referred to as the solid phase and fluid phase averages, respectively. ${ }^{6,9}$ The solid phase and fluid phase averages of $\xi$ are defined as

$$
\begin{aligned}
& \phi(\boldsymbol{x})\langle\xi\rangle^{\mathrm{s}}(\boldsymbol{x})=\int \chi(\boldsymbol{y}) \xi(\boldsymbol{y}) g(\boldsymbol{x}, \boldsymbol{y}) \mathrm{d} V, \\
& (1-\phi(\boldsymbol{x}))\langle\xi\rangle^{\mathrm{f}}(\boldsymbol{x})=\int(1-\chi(\boldsymbol{y})) \xi(\boldsymbol{y}) g(\boldsymbol{x}, \boldsymbol{y}) \mathrm{d} V .
\end{aligned}
$$

It is evident that the suspension average is related to the solid and fluid phase averages as

$$
\langle\xi\rangle=\phi\langle\xi\rangle^{\mathrm{s}}+(1-\phi)\langle\xi\rangle^{\mathrm{f}} .
$$

Another average that we will find useful is the particle phase average, ${ }^{6,9}$ defined as

$$
n(\boldsymbol{x})\langle\xi\rangle^{\mathrm{p}}=\sum_{i} \xi_{i} g_{i}
$$

where $\xi_{i} \equiv \xi\left(\boldsymbol{y}_{i}\right)$ and $g_{i} \equiv g\left(\boldsymbol{x}, \boldsymbol{y}_{i}\right), \boldsymbol{y}_{i}$ is the center of particle $i$, and the sum is over all particles in the entire volume $V$. For nonspherical particles, $\boldsymbol{y}_{i}$ is the center of volume, defined as $v^{\mathrm{p}} \boldsymbol{y}_{i}=\int_{V_{i}} \boldsymbol{y} \mathrm{d} V$, where $v^{\mathrm{p}}$ is the particle volume. It is evident from Eqs. (12) and (15) that $\langle\xi\rangle^{\mathrm{p}}$ is equal to $\langle\xi\rangle^{\mathrm{s}}$ when $\xi$ is defined only at the particle centers, i.e., $\xi=\xi_{i} v^{\mathrm{p}} \delta\left(\boldsymbol{y}-\boldsymbol{y}_{i}\right)$ within particle $i$.

The volume-averaged equations of motion are determined by volume averaging the pointwise balances of mass and momentum,

$$
\begin{aligned}
\hat{\boldsymbol{\nabla}} \cdot \boldsymbol{u} & =0 \\
\rho \frac{\hat{\mathrm{D}} \boldsymbol{u}}{\hat{\mathrm{D}} t} & =\hat{\boldsymbol{\nabla}} \cdot \boldsymbol{\sigma}+\boldsymbol{b},
\end{aligned}
$$

which apply at every point $\boldsymbol{y}$ in both the phases. For the rest of this section, we shall assume that particle and fluid inertia are negligibly small; this is primarily for ease of exposition, but it is indeed the case in Stokesian suspensions. As noted in $\S$, the main purpose of this paper is to derive expressions for the hydrodynamic part of the particle phase stress and the 
interphase drag, neither of which are affected by inertia. We shall therefore replace Eq. (17) by

$$
0=\hat{\boldsymbol{\nabla}} \cdot \boldsymbol{\sigma}+\boldsymbol{b} .
$$

to derive the volume-averaged momentum balances. The full momentum balances including inertia are provided in Appendix A. We ignore the balance of energy, under the assumption that changes in the temperature are not significant enough to alter the properties of the fluid and particles.

We first obtain the solid phase mass balance by multiplying Eq. (16) by $\chi g$ (here and hereafter, we have dropped the arguments of $\chi$ and $g$ for the sake of compactness) and integrating over the entire volume,

$$
\int(\hat{\boldsymbol{\nabla}} \cdot \boldsymbol{u}) \chi g \mathrm{~d} V=0,
$$

which may be written as

$$
\int \hat{\boldsymbol{\nabla}} \cdot(\boldsymbol{u} \chi g) \mathrm{d} V-\int g \boldsymbol{u} \cdot \hat{\boldsymbol{\nabla}} \chi \mathrm{d} V-\int \chi \boldsymbol{u} \cdot \hat{\boldsymbol{\nabla}} g \mathrm{~d} V=0 .
$$

By the application of the divergence theorem, the first integral on the left- hand side reduces to an integral over the surface $S$ that bounds the volume $V$, which in turn vanishes as a result of Eq. (7), provided the distance between the nearest point on the boundary and $\boldsymbol{x}$ is sufficiently large. Using Eqs. (6) and (3), and the fact that all the factors in the integrand of the third term except $g$ are not functions of $\boldsymbol{x}$, Eq. (20) transforms to

$$
\frac{\partial \phi}{\partial t}+\nabla \cdot\left(\phi\langle\boldsymbol{u}\rangle^{\mathrm{s}}\right)=0
$$

As already stated, $\boldsymbol{\nabla}$ is the gradient operator with respect to $\boldsymbol{x}$. Similarly, multiplying Eq. (16) by $(1-\chi) g$ and integrating over the entire volume, we obtain the fluid phase mass balance

$$
\frac{\partial(1-\phi)}{\partial t}+\nabla \cdot\left((1-\phi)\langle\boldsymbol{u}\rangle^{\mathrm{f}}\right)=0 .
$$

Summing Eqs. (21) and (22) and using Eq. (14), we get the mass balance for the suspension,

$$
\boldsymbol{\nabla} \cdot\langle\boldsymbol{u}\rangle=0 .
$$

Thus the suspension is incompressible, as the fluid and particles are individually incompressible. This does not, of course, mean that the suspension density $\langle\rho\rangle=\phi \rho^{\mathrm{p}}+(1$ $-\phi) \rho^{\mathrm{f}}$ is constant; an equation for the suspension density is readily obtained by taking the sum of $\rho^{\mathrm{p}}$ times Eq. (21) and $\rho^{\mathrm{f}}$ times Eq. (22), yielding

$$
\frac{\partial\langle\rho\rangle}{\partial t}+\nabla \cdot\left(\langle\rho\rangle\langle\boldsymbol{u}\rangle_{\mathrm{m}}\right)=0,
$$

where $\langle\boldsymbol{u}\rangle_{\mathrm{m}}$ is the mass-averaged suspension velocity, defined by

$$
\langle\rho\rangle\langle\boldsymbol{u}\rangle_{\mathrm{m}}=\phi \rho^{\mathrm{p}}\langle\boldsymbol{u}\rangle^{\mathrm{s}}+(1-\phi) \rho^{\mathrm{f}}\langle\boldsymbol{u}\rangle^{\mathrm{f}} .
$$

From the above discussion, it is clear that volumeaveraged equations may be written for either the solid and fluid phases, or for the entire suspension and one of the phases - the two sets are entirely equivalent, and which to use is a matter of convenience. ${ }^{6}$ Previous studies on suspension mechanics have tended to write balances for the suspension and the particle phase, and we too follow this convention. However, we also derive the fluid phase momentum balance, in order to illustrate the nature of the stress in each phase. (The relation between the momentum balances of the particle and solid phases will be revealed soon.)

The momentum balances of the suspension and the two phases (in the absence of inertia) are obtained by suitable averaging of Eq. (18). Multiplying it by $g$ and integrating over the entire volume, we obtain the suspension momentum balance

$$
0=\int g \hat{\boldsymbol{\nabla}} \cdot \boldsymbol{\sigma} \mathrm{d} V+\int g \boldsymbol{b} \mathrm{d} V
$$

which may be written as

$$
0=\int \hat{\boldsymbol{\nabla}} \cdot(g \boldsymbol{\sigma}) \mathrm{d} V-\int \hat{\boldsymbol{\nabla}} g \cdot \boldsymbol{\sigma} \mathrm{d} V+\int g \boldsymbol{b} \mathrm{d} V .
$$

The first integral vanishes on application of the divergence theorem and using Eq. (7). Using Eq. (6), we can then reduce Eq. (27) to the form

$$
0=\boldsymbol{\nabla} \cdot \int g \boldsymbol{\sigma} \mathrm{d} V+\langle\boldsymbol{b}\rangle .
$$

The integral in Eq. (28) is the suspension stress $\langle\boldsymbol{\sigma}\rangle$. To derive an expression for $\langle\boldsymbol{\sigma}\rangle$, we split the integral in Eq. (28) into integrals over the solid and fluid regions. We then obtain

$$
\begin{aligned}
0 & =\boldsymbol{\nabla} \cdot \int(1-\chi) g \boldsymbol{\sigma} \mathrm{d} V+\boldsymbol{\nabla} \cdot \int \chi g \boldsymbol{\sigma} \mathrm{d} V+\langle\boldsymbol{b}\rangle \\
& =\boldsymbol{\nabla} \cdot\left[(1-\phi)\langle\boldsymbol{\sigma}\rangle^{\mathrm{f}}+\boldsymbol{\phi}\langle\boldsymbol{\sigma}\rangle^{\mathrm{s}}\right]+\langle\boldsymbol{b}\rangle .
\end{aligned}
$$

We now determine expressions for the volume-averaged stress in the fluid and solid phases. If the fluid is Newtonian, $\langle\boldsymbol{\sigma}\rangle^{\mathrm{f}}$ is given by

$$
\begin{aligned}
(1-\phi)\langle\boldsymbol{\sigma}\rangle^{\mathrm{f}} & =\int_{V^{\mathrm{f}}}(-p \boldsymbol{I}+2 \eta \boldsymbol{e}) \mathrm{d} V \\
& =-(1-\phi)\langle p\rangle^{\mathrm{f}} \boldsymbol{I}+\int_{V} 2 \eta \boldsymbol{e} \mathrm{d} V \\
& =-(1-\phi)\langle p\rangle^{\mathrm{f}} \boldsymbol{I}+2 \eta\langle\boldsymbol{e}\rangle,
\end{aligned}
$$

where we have used the fact that the strain rate within the rigid particles vanishes. The rigidity of each particle also allows the stress within it to be written in terms of the surface traction and the body and inertial forces. To take advantage of this, we first expand $g$ within particle $i$ in a Taylor series about its center $\boldsymbol{y}_{i}$, 


$$
\begin{aligned}
g(\boldsymbol{x}, \boldsymbol{y})= & g\left(\boldsymbol{x}, \boldsymbol{y}_{i}\right)+\left.\boldsymbol{y}^{\prime} \cdot \hat{\boldsymbol{\nabla}} g(\boldsymbol{x}, \boldsymbol{y})\right|_{y_{i}}+\frac{1}{2 !} \boldsymbol{y}^{\prime} \boldsymbol{y}^{\prime}:\left.\hat{\boldsymbol{\nabla}} \hat{\boldsymbol{\nabla}} g(\boldsymbol{x}, \boldsymbol{y})\right|_{y_{i}} \\
& +\cdots
\end{aligned}
$$

where $\boldsymbol{y}^{\prime} \equiv \boldsymbol{y}-\boldsymbol{y}_{i}$. We remind the reader that $g_{i} \equiv g\left(\boldsymbol{x}, \boldsymbol{y}_{i}\right)$ is the weighting function at the center of particle $i$. Using the above expansion and Eq. (6), $\langle\boldsymbol{\sigma}\rangle^{\mathrm{s}}$ may be expressed as

$$
\phi\langle\boldsymbol{\sigma}\rangle^{\mathrm{s}}=\sum_{i} g_{i} \int_{V_{i}} \boldsymbol{\sigma} \mathrm{d} V-\boldsymbol{\nabla} \cdot \sum_{i} g_{i} \int_{V_{i}} \boldsymbol{y}^{\prime} \boldsymbol{\sigma} \mathrm{d} V+\cdots .
$$

We are now left with integrals of the stress and its moments over the volumes of the particle. These may be transformed to surface moments of the traction and volume moments of the body and inertia forces. As an example, consider the first term on the right-hand side of Eq. (32); this can be transformed using the identity

$$
\sigma=\left[\hat{\boldsymbol{\nabla}} \cdot\left(\sigma \boldsymbol{y}^{\prime}\right)\right]^{\mathrm{T}}-\boldsymbol{y}^{\prime} \hat{\boldsymbol{\nabla}} \cdot \sigma,
$$

a device employed by Batchelor. ${ }^{3}$ Using the divergence theorem for the integral of the first term, and substituting for $\hat{\boldsymbol{\nabla}} \cdot \boldsymbol{\sigma}$ from Eq. (18) in the second, we have

$$
\sum_{i} g_{i} \int_{V_{i}} \boldsymbol{\sigma} \mathrm{d} V=\sum_{i} g_{i} \int_{S_{i}} \boldsymbol{y}^{\prime} \boldsymbol{n} \cdot \boldsymbol{\sigma} \mathrm{d} S+\sum_{i} g_{i} \int_{V_{i}} \boldsymbol{y}^{\prime} \boldsymbol{b} \mathrm{d} V,
$$

Such a transformation exists for every term in the series in Eq. (32), and is given by Eq. (B5) in Appendix B. Substituting Eq. (34) and the similarly simplified forms of the higherorder terms in Eq. (32), we get

$$
\begin{aligned}
\boldsymbol{\phi}\langle\boldsymbol{\sigma}\rangle^{\mathrm{s}}= & \sum_{i} g_{i} \boldsymbol{S}_{i}-\frac{1}{2} \nabla \cdot \sum_{i} g_{i} \boldsymbol{Q}_{i}+\cdots \\
& +\sum_{i} g_{i} \int_{V_{i}} \boldsymbol{y}^{\prime} \boldsymbol{b} \mathrm{d} V-\frac{1}{2} \boldsymbol{\nabla} \cdot \sum_{i} g_{i} \int_{V_{i}} \boldsymbol{y}^{\prime} \boldsymbol{y}^{\prime} \boldsymbol{b} \mathrm{d} V+\cdots,
\end{aligned}
$$

were we have denoted the moments of the surface traction on particle $i$ as

$$
\begin{aligned}
\boldsymbol{f}_{i} & =\int_{S_{i}} \boldsymbol{n} \cdot \boldsymbol{\sigma} \mathrm{d} S, \quad \boldsymbol{S}_{i}=\int_{S_{i}} \boldsymbol{y}^{\prime} \boldsymbol{n} \cdot \boldsymbol{\sigma} \mathrm{d} S, \\
\boldsymbol{Q}_{i} & =\int_{S_{i}} \boldsymbol{y}^{\prime} \boldsymbol{y}^{\prime} \boldsymbol{n} \cdot \boldsymbol{\sigma} \mathrm{d} S, \cdots .
\end{aligned}
$$

In Eq. (35), the terms in the first line arise from the moments of the surface traction, namely the first term on the righthand-side of Eq. (34) and the first term in Eq. (B5); the subsequent terms arise from the volume moments of the body forces, namely the second term on the right-hand-side of Eq. (34) and the second term in Eq. (B5). The zeroth moment of the surface traction is the monopole $f_{i}$, or force on the particle, the first moment $\boldsymbol{S}_{i}$ is the dipole, the second moment $\boldsymbol{Q}_{i}$ is the quadrupole, and so on. Surface traction is due to hydrodynamic forces exerted by the fluid, and the forces due to particle contact. Hence we may write the moments as

$$
f_{i}=f_{i}^{\mathrm{h}}+f_{i}^{\mathrm{c}}, \quad S_{i}=S_{i}^{\mathrm{h}}+S_{i}^{\mathrm{c}}, \quad Q_{i}=Q_{i}^{\mathrm{h}}+Q_{i}^{\mathrm{c}}, \cdots,
$$

the superscripts $h$ and $c$ indicating the parts arising from hydrodynamic and contact traction, respectively.

A slight simplification of Eq. (35) may be effected by writing the body force density in particle $i$ as the sum of its mean $\boldsymbol{b}_{i}$ and a deviation $\boldsymbol{b}^{\prime}$. Using the equations of linear and angular momentum for the particle

$$
0=\boldsymbol{f}_{i}+\boldsymbol{b}_{i} v^{\mathrm{p}}, \quad 0=-\boldsymbol{\epsilon}: \int_{S_{i}} \boldsymbol{y}^{\prime}(\boldsymbol{n} \cdot \boldsymbol{\sigma}) \mathrm{d} S+\boldsymbol{\tau}_{i},
$$

where $\tau_{i}$ is the external torque on particle $i$ and $\epsilon$ is the alternating tensor, and the identities $\int y^{\prime} \mathrm{d} V=0$ and $\int_{V_{i}} \boldsymbol{y}^{\prime} \boldsymbol{y}^{\prime} \rho \mathrm{d} V=(1 / 5) \rho^{\mathrm{p}} v^{\mathrm{p}} a^{2} \boldsymbol{I}\left(v^{\mathrm{p}}\right.$ being is the particle volume as defined earlier), the expression for the solid phase stress tensor becomes

$$
\begin{aligned}
\phi\langle\boldsymbol{\sigma}\rangle^{\mathrm{s}}= & \sum_{i} g_{i}\left(\overline{\boldsymbol{S}}_{i}-\frac{1}{2} \boldsymbol{\epsilon} \cdot \boldsymbol{\tau}_{i}+\int_{V_{i}} \boldsymbol{y}^{\prime} \boldsymbol{b}^{\prime} \mathrm{d} V\right) \\
& -\frac{1}{2} \boldsymbol{\nabla} \cdot \sum_{i} g_{i}\left(\boldsymbol{Q}_{i}-\frac{1}{5} v^{\mathrm{p}} a^{2} \boldsymbol{I} \boldsymbol{f}_{i}+\int_{V_{i}} \boldsymbol{y}^{\prime} \boldsymbol{y}^{\prime} \boldsymbol{b}^{\prime} \mathrm{d} V\right) \\
& +\cdots .
\end{aligned}
$$

Here $\bar{S}_{i} \equiv\left(\frac{1}{2}\right)\left(S_{i}+S_{i}^{\mathrm{T}}\right)$ is the symmetric part of the dipole, usually called the stresslet.

Substituting Eq. (30) in Eq. (29), the momentum balance for the suspension takes the form

$$
0=\langle\boldsymbol{b}\rangle-\nabla\left[(1-\phi)\langle p\rangle^{\mathrm{f}}\right]+2 \eta \boldsymbol{\nabla} \cdot\langle\boldsymbol{e}\rangle+\nabla \cdot\left(\phi\langle\boldsymbol{\sigma}\rangle^{\mathrm{s}}\right),
$$

with $\langle\boldsymbol{\sigma}\rangle^{\mathrm{s}}$ given by Eq. (39). Thus, the "particle stress" $\boldsymbol{\Sigma}^{(\mathrm{p})}$, introduced by Batchelor ${ }^{3}$ as the particle contribution to the suspension stress, is nothing but $\phi\langle\boldsymbol{\sigma}\rangle^{\mathrm{s}}$; when intertial effects are included, there is an additional contribution to $\boldsymbol{\Sigma}^{(\mathrm{p})}$ in the form of the Reynolds's stress (see Appendix A). Apart from the inertial terms, the above expression for $\phi\langle\boldsymbol{\sigma}\rangle^{\mathrm{s}}$ differs in one significant way from Batchelor's expression for $\boldsymbol{\Sigma}^{(\mathrm{p})}$ : Batchelor restricted attention to a uniform stress state, whence his expression for $\mathbf{\Sigma}^{(p)}$ does not contain the higherorder terms that involve gradients, but we do not make this restriction. The importance of the higher-order terms grows as the state of the suspension becomes increasingly nonuniform. Thus, the relation $\boldsymbol{\Sigma}^{(\mathrm{p})}=n\langle S\rangle^{\mathrm{p}}$, which has been used in many studies as a general expression for the stress in a Stokesian suspension, is actually only the first term in a gradient expansion.

Next, we determine the momentum balance for the solid phase by multiplying Eq. (18) by $\chi g$ and integrating over the entire volume. Using Eqs. (3), (4), and (16), and employing the same manipulations as in Eq. (27), we get

$$
0=\boldsymbol{\nabla} \cdot\left(\phi\langle\boldsymbol{\sigma}\rangle^{\mathrm{s}}\right)+\sum_{i} \int_{S_{i}} \boldsymbol{n} \cdot \boldsymbol{\sigma} g \mathrm{~d} S+\phi\langle\boldsymbol{b}\rangle^{\mathrm{s}} .
$$

The second term on the right-hand side is clearly the volume-averaged traction on the particle surfaces. To evalu- 
ate the integral, we substitute the Taylor expansion for $g$ from Eq. (31); the result is

$$
\begin{aligned}
\sum_{i} \int_{S_{i}} \boldsymbol{n} \cdot \boldsymbol{\sigma} g \mathrm{~d} S= & \sum_{i} g_{i} \boldsymbol{f}_{i}-\boldsymbol{\nabla} \cdot\left[\sum_{i} g_{i} \boldsymbol{S}_{i}\right. \\
& \left.-\frac{1}{2} \boldsymbol{\nabla} \cdot \sum_{i} g_{i} \boldsymbol{Q}_{i}+\cdots\right]
\end{aligned}
$$

On substituting in Eq. (41) the expansions for $\boldsymbol{\nabla} \cdot\left(\phi\langle\boldsymbol{\sigma}\rangle^{\mathrm{s}}\right)$ from Eq. (35) and $\Sigma_{i} \int_{S_{i} \boldsymbol{n} \cdot \boldsymbol{\sigma} g} \mathrm{~d} S$ from Eq. (42), it is immediately clear that all the terms in the latter, except the first, are cancelled by the terms in the first line of the former. With the cancellations, the solid phase momentum balance reduces to

$$
\begin{aligned}
0= & \phi\langle\boldsymbol{b}\rangle^{\mathrm{s}}+\sum_{i} g_{i} \boldsymbol{f}_{i}+\boldsymbol{\nabla} \cdot\left[\sum_{i} g_{i} \int_{V_{i}} \boldsymbol{y}^{\prime} \boldsymbol{b} \mathrm{d} V\right. \\
& \left.-\frac{1}{2} \boldsymbol{\nabla} \cdot \sum_{i} g_{i} \int_{V_{i}} \boldsymbol{y}^{\prime} \boldsymbol{y}^{\prime} \boldsymbol{b} \mathrm{d} V+\cdots\right]
\end{aligned}
$$

Nott and Brady ${ }^{2}$ erred in assuming that the volume-averaged traction is the particle-averaged force, i.e., the first term in Eq. (42); as a result, they did not realize the cancellation of terms mentioned above, and retained $\boldsymbol{\nabla} \cdot\left(\phi\langle\boldsymbol{\sigma}\rangle^{\mathrm{s}}\right)$ in Eq. (43).

Though Eq. (43) is adequate, it can be simplified considerably in the following way. As noted earlier, the terms within the square brackets are volume moments about the particle centers of $\boldsymbol{b}$, starting from the first moment. The volume-averaged body force $\phi\langle\boldsymbol{b}\rangle^{\mathrm{s}}$ may also be written as the sum of moments, by replacing $g$ with its Taylor expansion [Eq. (31)]. It is immediately apparent that all moments, except the zeroth, cancel exactly with the terms in the square brackets, yielding

$$
0=v^{\mathrm{p}} \sum_{i} g_{i} \boldsymbol{b}_{i}+\sum_{i} g_{i} \boldsymbol{f}_{i}
$$

It is useful to decompose the net body force on each particle $v^{\mathrm{p}} \boldsymbol{b}_{i}$ into an external force $v^{\mathrm{p}} \boldsymbol{b}_{i}^{\text {ext }}$ (such as gravity) and an "action at a distance" interparticle force $f_{i}^{\mathrm{ip}}$. This reduces Eq. (44) to

$$
0=v^{\mathrm{p}} \sum_{i} g_{i} \boldsymbol{b}_{i}^{\mathrm{ext}}+\sum_{i} g_{i}\left(\boldsymbol{f}_{i}+\boldsymbol{f}_{i}^{\mathrm{ip}}\right) .
$$

Both the terms in this equation are particle phase averages [defined in Eq. (15)], whence it may be written as

$$
0=n v^{\mathrm{p}}\left\langle\boldsymbol{b}^{\mathrm{ext}}\right\rangle^{\mathrm{p}}+n\langle f\rangle^{\mathrm{p}},
$$

where $\langle f\rangle^{\mathrm{p}} \equiv\left\langle f^{\mathrm{h}}\right\rangle^{\mathrm{p}}+\left\langle f^{\mathrm{c}}\right\rangle^{\mathrm{p}}+\left\langle f^{\mathrm{i}}\right\rangle^{\mathrm{p}}$ is the net force on the particle phase due to hydrodynamic and contact traction forces, and the action at a distance interparticle force. This is the momentum balance for the particle phase in the absence of inertia. Jackson ${ }^{6}$ and Prosperetti ${ }^{8}$ indicated that the solid phase and particle phase momentum balances are equal up to $\mathrm{O}\left(a^{2} / L^{2}\right)$-we have shown above that the equality is exact. The latter is simply another form of the former. The utility of Eq. (46), apart from its simpler and more compact form, is that particle phase averages are accessed much more easily in experiments and particle dynamics simulations.
Anderson and Jackson ${ }^{9}$ and Jackson ${ }^{6}$ derived Eq. (46) in a simpler and more direct manner, by multiplying the first of Eq. (38) by $g_{i}$ and summing over all $i$. Our motivation for arriving at the particle phase momentum balance via the solid phase momentum balance is to show that they are just different forms of the same balance, and that the stresslet is absent in both. This contradicts the assumption of Nott and Brady $^{2}$ and the ensuing studies that used or extended the $\mathrm{SBM}^{19-24}$ that the particle phase stress derives from the stresslet.

Equation (46) poses a dilemma: if there is no term of hydrodynamic origin that has the form of the divergence of a stress, how is particle migration to be accounted? (Recall from §I that particle migration is driven by the divergence of $\Sigma^{\mathrm{h}(\mathrm{p})}$ in the suspension balance model.) This question is answered in the following section, where we show that force on the particle phase, $n\langle f\rangle^{\mathrm{p}}$, can be written as the sum of an interphase drag $n\left\langle f^{\mathrm{h}}\right\rangle_{\text {drag }}$ and the divergence of a particle phase stress $n\langle\boldsymbol{\sigma}\rangle^{\mathrm{p}}$. We argue that the latter provides the driving force for migration at lowest order in the gradients.

From the definition of the volume averages, it is obvious that the momentum balance for the fluid phase is the difference between the momentum balances for the suspension [Eq. (40)] and the solid phase [Eq. (43)]. Thus, any two of these balances suffice to describe the two-phase system, as noted earlier. Nevertheless, it is useful to study the momentum balance for the fluid phase. Multiplying Eq. (18) by $(1-\chi) g$ and integrating over the entire volume, we get

$$
0=\boldsymbol{\nabla} \cdot\left[(1-\phi)\langle\boldsymbol{\sigma}\rangle^{\mathrm{f}}\right]-\sum_{i} \int_{S_{i}} \boldsymbol{n} \cdot \boldsymbol{\sigma}^{\mathrm{f}} g \mathrm{~d} S+(1-\phi)\langle\boldsymbol{b}\rangle^{\mathrm{f}} .
$$

Note that the surface integral in Eq. (47) represents the volume average of only the fluid traction on the particle surfaces-the contact traction is not present, unlike in the particle phase momentum balance Eq. (41). Substituting Eqs. (30) and (42) in Eq. (47) yields

$$
\begin{aligned}
0= & (1-\phi)\langle\boldsymbol{b}\rangle^{\mathrm{f}}-n\left\langle\boldsymbol{f}^{\mathrm{h}}\right\rangle^{\mathrm{p}}-\boldsymbol{\nabla}\left[(1-\phi)\langle p\rangle^{\mathrm{f}}\right]+2 \eta \boldsymbol{\nabla} \cdot\langle\boldsymbol{e}\rangle \\
& +\boldsymbol{\nabla} \cdot\left[n\left\langle\boldsymbol{S}^{\mathrm{h}}\right\rangle^{\mathrm{p}}-\frac{1}{2} \boldsymbol{\nabla} \cdot\left(n\left\langle\boldsymbol{Q}^{\mathrm{h}}\right\rangle^{\mathrm{p}}\right)+\cdots\right] .
\end{aligned}
$$

The quantity within the square brackets is $\Sigma^{\mathrm{h}(\mathrm{p})}$, the hydrodynamic part of the "particle stress" $\boldsymbol{\Sigma}^{(\mathrm{p})}$ [see Eq. (35)]. The hydrodynamic force on the particle phase $n\left\langle f^{\mathrm{h}}\right\rangle^{\mathrm{p}}$ appears here too, but with sign opposite to that in Eq. (46), in accord with Newton's third law. Using the decomposition of $n\left\langle f^{\mathrm{h}}\right\rangle \mathrm{p}$ derived in $\S I I I B$, we see that the net stress in the fluid phase momentum balance is $(1-\phi)\langle\boldsymbol{\sigma}\rangle^{\mathrm{f}}+\boldsymbol{\Sigma}^{\mathrm{h}(\mathrm{p})}-n\left\langle\boldsymbol{\sigma}^{\mathrm{h}}\right\rangle$.

At this point, a few comments on the volume-averaged equations of motion are in order. We have derived the equations by a formal averaging process, with the only assumption that the particles are spherical and rigid. The assumption of spherical particles too may be relaxed if the center of each particle identified as its center of volume. The volumeaveraged stress in the fluid phase, $(1-\phi)\langle\boldsymbol{\sigma}\rangle^{\mathrm{f}}$, is purely Newtonian if the fluid is Newtonian [see Eq. (30)], and the volume-averaged stress in the solid phase $\phi\langle\boldsymbol{\sigma}\rangle^{\mathrm{s}}$ is in essence the "particle stress" introduced by Batchelor ${ }^{3}$ [see Eqs. (40) 
and (41)]. However, the net stresses appearing in the balances of momentum for the two phases are not $(1-\phi)\langle\boldsymbol{\sigma}\rangle^{\mathrm{f}}$ and $\phi\langle\boldsymbol{\sigma}\rangle^{\mathrm{s}}$, respectively. This is because the volume- averaged traction at the surfaces of the particles is the sum of a particle-averaged force and the divergence of a stress, as shown in Eq. (42). In the solid phase, this extra stress cancels with a part of $\phi\langle\boldsymbol{\sigma}\rangle^{\text {s }}$, leaving behind only the particleaveraged force, as shown in Eq. (46). In the fluid phase, this extra stress contributes the quantity $\boldsymbol{\Sigma}^{\mathrm{h}(\mathrm{p})}$ to the net stress, as shown in Eq. (48). Further, the following section shows that the particle-averaged force can be decomposed to the sum of an interphase drag and the divergence of a stress. Indeed, exact calculations ${ }^{6,7}$ for dilute suspensions of Stokesian particles show the effective viscosity of the fluid phase to be enhanced by the familiar Einstein correction. Similarly, analysis of the flow through dilute random array of spheres yields the Brinkman equation for the fluid-phase, with an effective viscosity exceeding that of the pure fluid. ${ }^{28}$

\section{PARTICLE PHASE STRESS AND INTERPHASE DRAG IN A STOKESIAN SUSPENSION}

We shall now demonstrate that the force on the particle phase $n\langle f\rangle^{\mathrm{p}}$ may be written as the sum of the interphase drag and the divergence of a stress. This is easier to appreciate if we first consider the average of a pairwise additive interparticle force, such as the contact force, the result for which is already known. This result then leads naturally to the relation for the average hydrodynamic force.

\section{A. Stress due to nonhydrodynamic interparticle forces}

We first consider the average contact force $\left\langle f^{c}\right\rangle^{p}$. Assuming that these forces are pairwise additive, and denoting the force on particle $i$ due to particle $j(i \neq j)$ as $f_{i j}^{\mathrm{c}}$, we have

$$
n\left\langle\boldsymbol{f}^{\mathrm{c}}\right\rangle^{\mathrm{p}}=\sum_{i} g_{i} \sum_{j \neq i} \boldsymbol{f}_{i j}^{\mathrm{c}}
$$

which may be written as ${ }^{6}$

$$
n\left\langle\boldsymbol{f}^{\mathrm{c}}\right\rangle^{\mathrm{p}}=\sum_{i} \sum_{j \neq i} g_{i} \boldsymbol{f}_{i j}^{\mathrm{c}}-\sum_{i} \sum_{j \neq i} g_{i j}^{\mathrm{c}} f_{i j}^{\mathrm{c}}
$$

because the second double sum vanishes identically as a consequence of Newton's third law, $\boldsymbol{f}_{i j}^{\mathrm{c}}=-\boldsymbol{f}_{j i}^{\mathrm{c}}$. Here we have used the notation $g_{i j}^{\mathrm{c}} \equiv g\left(\boldsymbol{x}, \boldsymbol{y}_{i j}^{\mathrm{c}}\right)$, where $\boldsymbol{y}_{i j}^{\mathrm{c}}$ is the point of contact of particles $i$ and $j$. We expand $g_{i j}^{\mathrm{c}}$ in a Taylor series about $g_{i}$,

$$
\begin{aligned}
g_{i j}^{\mathrm{c}}= & g_{i}+\left.\left(\boldsymbol{y}_{i j}^{\mathrm{c}}-\boldsymbol{y}_{i}\right) \cdot \hat{\boldsymbol{\nabla}} g(\boldsymbol{x}, \boldsymbol{y})\right|_{\boldsymbol{y}_{i}} \\
& +\frac{1}{2}\left(\boldsymbol{y}_{i j}^{\mathrm{c}}-\boldsymbol{y}_{i}\right)\left(\boldsymbol{y}_{i j}^{\mathrm{c}}-\boldsymbol{y}_{i}\right):\left.\hat{\boldsymbol{\nabla}} \hat{\nabla} g(\boldsymbol{x}, \boldsymbol{y})\right|_{\boldsymbol{y}_{i}}+\cdots,
\end{aligned}
$$

and substitute in Eq. (50). Using Eq. (6), we finally get

$$
n\left\langle\boldsymbol{f}^{\mathrm{c}}\right\rangle^{\mathrm{p}}=\boldsymbol{\nabla} \cdot\left(n\left\langle\boldsymbol{\sigma}^{\mathrm{c}}\right\rangle^{\mathrm{p}}\right),
$$

where

$$
\begin{aligned}
n\left\langle\boldsymbol{\sigma}^{\mathrm{c}}\right\rangle^{\mathrm{p}}= & \sum_{i} g_{i} \sum_{j \neq i}\left(\boldsymbol{y}_{i j}^{\mathrm{c}}-\boldsymbol{y}_{i}\right) \boldsymbol{f}_{i j}^{\mathrm{c}} \\
& -\frac{1}{2} \boldsymbol{\nabla} \cdot \sum_{i} g_{i} \sum_{j \neq i}\left(\boldsymbol{y}_{i j}^{\mathrm{c}}-\boldsymbol{y}_{i}\right)\left(\boldsymbol{y}_{i j}^{\mathrm{c}}-\boldsymbol{y}_{i}\right) \boldsymbol{f}_{i j}^{\mathrm{c}}+\cdots \\
= & \sum_{i} g_{i} \boldsymbol{S}_{i}^{\mathrm{c}}-\frac{1}{2} \boldsymbol{\nabla} \cdot \sum_{i} g_{i} \boldsymbol{Q}_{i}^{\mathrm{c}}+\cdots
\end{aligned}
$$

The latter equality follows from the definition of the moments Eq. (36) for forces acting at discrete points on the particle surface. Thus, the volume-averaged contact force can be written as the divergence of a stress. The first term in Eq. (53) is widely used to determine the contact stress in discrete element simulations of granular materials. As in the case of $\boldsymbol{\Sigma}^{(p)}$, the higher-order terms gain importance as the spatial non-uniformity of he stress increases.

The volume average of any action at a distance interparticle force $f^{\text {ip }}$ that is pairwise additive, such as the electrostatic force, may also be determined in the same manner. Unlike the contact force, there is no specific point of action, but the procedure used above requires only an intermediate point $\boldsymbol{y}_{i j}^{\mathrm{m}} \equiv \alpha \boldsymbol{y}_{i}+(1-\alpha) \boldsymbol{y}_{j}$, such that $g_{i j}^{\mathrm{m}}=g_{j i}^{\mathrm{m}}$. By our assumption that $g(\boldsymbol{x}, \boldsymbol{y})$ depends only on the difference $\boldsymbol{y}-\boldsymbol{x}$, the above constraint implies $\boldsymbol{y}_{i j}^{\mathrm{m}}=\boldsymbol{y}_{j i}^{\mathrm{m}}$, and hence $\alpha=1 / 2$. Thus, the midpoint $\boldsymbol{y}_{i j}^{\mathrm{m}} \equiv(1 / 2)\left(\boldsymbol{y}_{i}+\boldsymbol{y}_{j}\right)$ between the centers of the pair of particles assumes the role of the point of contact for forces that act at a distance. Proceeding in the same manner as above, but with $\boldsymbol{y}_{i j}^{\mathrm{m}}$ replacing $\boldsymbol{y}_{i j}^{\mathrm{c}}$ it follows immediately that

$$
n\left\langle f^{\mathrm{i}\rangle}\right\rangle^{\mathrm{p}}=\boldsymbol{\nabla} \cdot\left(n\left\langle\boldsymbol{\sigma}^{\mathrm{ip}}\right\rangle^{\mathrm{p}}\right),
$$

where

$$
\begin{aligned}
n\left\langle\boldsymbol{\sigma}^{\mathrm{ip}}\right\rangle^{\mathrm{p}}= & \sum_{i} g_{i} \sum_{j \neq i}\left(\boldsymbol{y}_{i j}^{\mathrm{m}}-\boldsymbol{y}_{i}\right) \boldsymbol{f}_{i j}^{\mathrm{ip}} \\
& -\frac{1}{2} \boldsymbol{\nabla} \cdot \sum_{i} g_{i} \sum_{j \neq i}\left(\boldsymbol{y}_{i j}^{\mathrm{m}}-\boldsymbol{y}_{i}\right)\left(\boldsymbol{y}_{i j}^{\mathrm{m}}-\boldsymbol{y}_{i}\right) \boldsymbol{f}_{i j}^{\mathrm{ip}}+\cdots \\
= & \sum_{i} g_{i} \boldsymbol{S}_{i}^{\mathrm{ip}}-\frac{1}{2} \boldsymbol{\nabla} \cdot \sum_{i} g_{i} \boldsymbol{Q}_{i}^{\mathrm{ip}}+\cdots
\end{aligned}
$$

If the inter-particle forces are not pairwise additive, but if the sum of the forces on all the particles is zero, it is easily shown $^{29}$ that the stress $\left\langle\boldsymbol{\sigma}^{\mathrm{ip}}\right\rangle^{\mathrm{p}}$ may be written as

$$
\begin{aligned}
n\left\langle\boldsymbol{\sigma}^{\mathrm{i} \mathrm{p}}\right\rangle^{\mathrm{p}}= & -\sum_{i}\left(\left(\boldsymbol{y}_{i}-\boldsymbol{y}_{0}\right) g_{i}\right. \\
& \left.-\frac{1}{2} \boldsymbol{\nabla} \cdot\left(\boldsymbol{y}_{i}-\boldsymbol{y}_{0}\right)\left(\boldsymbol{y}_{i}-\boldsymbol{y}_{0}\right) g_{i}+\cdots\right) \boldsymbol{f}_{i},
\end{aligned}
$$

where $\boldsymbol{y}_{0}$ is an arbitrary reference point; the most convenient choice would be $\boldsymbol{y}_{0}=\boldsymbol{x}$. If the stress is spatially uniform, this reduces to the familiar form $-\left\langle\left(\boldsymbol{y}-\boldsymbol{y}_{0}\right) \boldsymbol{f}\right\rangle^{\mathrm{p}}{ }^{27,29}$ 


\section{B. Stress and interphase drag due to hydrodynamic forces}

Until this point, our analysis made no restrictions on the particle Reynolds number $\mathrm{Re}_{\mathrm{p}}$. Though the effects of particle and fluid inertia in were neglected in §II, it was only for ease of exposition; the full momentum balances given in Appendix A include the inertial contributions. However, we shall now restrict attention to vanishingly small fluid inertia, or $\operatorname{Re}_{\mathrm{p}} \ll 1$-we comment in $\S I V$ on how the procedure may be generalized to arbitrary $\mathrm{Re}_{\mathrm{p}}$.

The treatment of $\left\langle f^{\mathrm{h}}\right\rangle^{\mathrm{p}}$ is similar to that of $\left\langle f^{\mathrm{ip}\rangle \mathrm{p}}\right.$, but it must take account of the difference that the hydrodynamic forces are neither pairwise additive nor does their sum over all particles vanish. Nevertheless, we may make analytical progress by recognizing that the linearity of Stokes equations allows the force on particle $i$ to be expressed as ${ }^{30,31}$

$$
\begin{aligned}
\boldsymbol{f}_{i}^{\mathrm{h}}= & \sum_{j}\left[\boldsymbol{R}_{i j}^{\mathrm{FU}} \cdot\left(\boldsymbol{u}_{j}-\langle\boldsymbol{u}\rangle_{j}\right)+\boldsymbol{R}_{i j}^{\mathrm{F} \Omega} \cdot\left(\boldsymbol{\omega}_{j}-\langle\boldsymbol{\omega}\rangle_{j}\right)+\boldsymbol{R}_{i j}^{\mathrm{FE}}:\left(-\langle\boldsymbol{e}\rangle_{j}\right)\right. \\
& +\cdots]
\end{aligned}
$$

where $\boldsymbol{u}_{j}$ and $\boldsymbol{\omega}_{j}$ are the velocity and angular velocity of particle $j$, and $\langle\boldsymbol{u}\rangle_{j},\langle\boldsymbol{\omega}\rangle_{j}$ and $\langle\boldsymbol{e}\rangle_{j}$ are the velocity, half the vorticity, and the strain rate of the suspension at $\boldsymbol{x}=\boldsymbol{y}_{j}$. Also, $\boldsymbol{R}^{\mathrm{FU}}, \boldsymbol{R}^{\mathrm{F} \Omega}$, and $\boldsymbol{R}^{\mathrm{FE}}$ are the purely configuration dependent resistance tensors that relate the force to the velocity, angular velocity and strain rate, respectively. The expression for $f_{i j}^{\text {h }}$ [Eq. (57)] may be continued to any order in the gradient of $\langle\boldsymbol{u}\rangle$. For the sake of compactness, we write Eq. (57) as

$$
f_{i}^{\mathrm{h}}=\sum_{j} f_{i j}^{\mathrm{h}}
$$

where

$$
\begin{aligned}
\boldsymbol{f}_{i j}^{\mathrm{h}} \equiv & \boldsymbol{R}_{i j}^{\mathrm{FU}} \cdot\left(\boldsymbol{u}_{j}-\langle\boldsymbol{u}\rangle_{j}\right)+\boldsymbol{R}_{i j}^{\mathrm{F \Omega}} \cdot\left(\boldsymbol{\omega}_{j}-\langle\boldsymbol{\omega}\rangle_{j}\right)+\boldsymbol{R}_{i j}^{\mathrm{FE}}:\left(-\langle\boldsymbol{e}\rangle_{j}\right) \\
& +\cdots .
\end{aligned}
$$

Equation (58) does not imply pairwise additivity as it is normally understood, because (a) $\boldsymbol{R}_{i j}^{\mathrm{FU}}, \boldsymbol{R}_{i j}^{\mathrm{F} \Omega}$, and $\boldsymbol{R}_{i j}^{\mathrm{FE}}$ depend not only on the positions of particles $i$ and $j$, but of all the particles in the system, (b) $f_{i j}^{\mathrm{h}}$ is in general not related to $f_{j i}^{\mathrm{h}}$, and (c) $f_{i i}^{\mathrm{h}} \neq 0$, as the motion of a particle relative to the suspension around it causes a hydrodynamic force on itself. Nevertheless, Eq. (59) is a unique partition of the net force on a particle into pairwise components. We emphasize that the actual hydrodynamic force does not act at a distance, but on the surface of each particle. However, it is convenient to think of it as the sum of the interaction forces $f_{i j}^{\mathrm{h}}$; moreover, such a representation arises naturally in the microhydrodynamic analysis of Stokesian suspensions, as a result of the linearity of Stokes equations. ${ }^{32}$

The hydrodynamic force may now be written as

$$
n\left\langle\boldsymbol{f}^{\mathrm{h}}\right\rangle^{\mathrm{p}}=\sum_{i} g_{i} f_{i i}^{\mathrm{h}}+\sum_{i} g_{i} \sum_{j \neq i} \boldsymbol{f}_{i j}^{\mathrm{h}} .
$$

Following the procedure for the determination of $\left\langle f^{c}\right\rangle^{\mathrm{p}}$ and $\left\langle f^{\mathrm{ip}}\right\rangle^{\mathrm{p}}$ in §IIIA, we subtract the double sum $\Sigma_{i} \Sigma_{j \neq i} g_{i j}^{\mathrm{m}} f_{i j}^{\mathrm{h}}$ from the right-hand side of Eq. (60), where $g_{i j}^{\mathrm{m}} \equiv g\left(\boldsymbol{x}, \boldsymbol{y}_{i j}^{\mathrm{m}}\right)$, and $\boldsymbol{y}_{i j}^{\mathrm{m}} \equiv\left(\frac{1}{2}\right)\left(\boldsymbol{y}_{i}+\boldsymbol{y}_{j}\right)$. However, this double sum does not vanish identically, as $f_{i j}^{\mathrm{h}} \neq-f_{j i}^{\mathrm{h}}$, and hence we also add the same. The result is

$$
\begin{aligned}
n\left\langle f^{\mathrm{h}}\right\rangle^{\mathrm{p}}= & \sum_{i} g_{i} f_{i i}^{\mathrm{h}}+\sum_{i} \sum_{j \neq i} g_{i} f_{i j}^{\mathrm{h}}-\sum_{i} \sum_{j \neq i} g_{i j}^{\mathrm{m}} f_{i j}^{\mathrm{h}} \\
& +\sum_{i} \sum_{j \neq i} g_{i j}^{\mathrm{m}} f_{i j}^{\mathrm{h}}=\sum_{i} g_{i} f_{i i}^{\mathrm{h}}+\sum_{i} \sum_{j \neq i} g_{i j}^{\mathrm{m}} f_{i j}^{\mathrm{h}} \\
& +\sum_{i} \sum_{j \neq i}\left(g_{i}-g_{i j}^{\mathrm{m}}\right) f_{i j}^{\mathrm{h}}
\end{aligned}
$$

Substituting the Taylor expansion of $g_{i j}^{\mathrm{m}}$ about $g_{i}$ in Eq. (61), and using Eq. (6), we finally get

$$
n\left\langle f^{\mathrm{h}}\right\rangle^{\mathrm{p}}=n\left\langle f^{\mathrm{h}}\right\rangle_{\mathrm{drag}}+\boldsymbol{\nabla} \cdot\left(n\left\langle\boldsymbol{\sigma}^{\mathrm{h}}\right\rangle^{\mathrm{p}}\right),
$$

where $\left\langle f^{\mathrm{h}}\right\rangle_{\text {drag }}$ is the volume-averaged interphase drag, given by

$$
n\left\langle f^{\mathrm{h}}\right\rangle_{\mathrm{drag}}=\sum_{i} g_{i} f_{i i}^{\mathrm{h}}+\sum_{i} \sum_{j \neq i} g_{i j}^{\mathrm{m}} \boldsymbol{f}_{i j}^{\mathrm{h}},
$$

and $\left\langle\boldsymbol{\sigma}^{\mathrm{h}}\right\rangle^{\mathrm{p}}$ is the particle phase hydrodynamic stress, given by

$$
\begin{aligned}
n\left\langle\boldsymbol{\sigma}^{\mathrm{h}}\right\rangle^{\mathrm{p}}= & \sum_{i} g_{i} \sum_{j}\left(\boldsymbol{y}_{i j}^{\mathrm{m}}-\boldsymbol{y}_{i}\right) \boldsymbol{f}_{i j}^{\mathrm{h}}-\frac{1}{2} \boldsymbol{\nabla} \cdot \sum_{i} g_{i} \sum_{j}\left(\boldsymbol{y}_{i j}^{\mathrm{m}}-\boldsymbol{y}_{i}\right) \\
& \times\left(\boldsymbol{y}_{i j}^{\mathrm{m}}-\boldsymbol{y}_{i}\right) \boldsymbol{f}_{i j}^{\mathrm{h}}+\cdots
\end{aligned}
$$

In terms of the resistance functions, these relations take the form

$$
\begin{aligned}
n\left\langle\boldsymbol{f}^{\mathrm{h}}\right\rangle_{\mathrm{drag}}= & \sum_{i} \sum_{j} g_{i j}^{\mathrm{m}}\left[\boldsymbol{R}_{i j}^{\mathrm{FU}} \cdot\left(\boldsymbol{u}_{j}-\langle\boldsymbol{u}\rangle_{j}\right)+\boldsymbol{R}_{i j}^{\mathrm{F} \Omega} \cdot\left(\boldsymbol{\omega}{ }_{j}-\langle\boldsymbol{\omega}\rangle_{j}\right)\right. \\
& \left.-\boldsymbol{R}_{i j}^{\mathrm{FE}}:\langle\boldsymbol{e}\rangle_{j}+\cdots\right]
\end{aligned}
$$

and

$$
\begin{aligned}
n\left\langle\boldsymbol{\sigma}^{\mathrm{h}}\right\rangle^{\mathrm{p}}= & \sum_{i} g_{i} \sum_{j}\left(\boldsymbol{y}_{i j}^{\mathrm{m}}-\boldsymbol{y}_{i}\right)\left[\boldsymbol{R}_{i j}^{\mathrm{FU}} \cdot\left(\boldsymbol{u}_{j}-\langle\boldsymbol{u}\rangle_{j}\right)+\boldsymbol{R}_{i j}^{\mathrm{F} \Omega}\right. \\
& \left.\cdot\left(\boldsymbol{\omega}_{j}-\langle\boldsymbol{\omega}\rangle_{j}\right)-\boldsymbol{R}_{i j}^{\mathrm{FE}}:\langle\boldsymbol{e}\rangle_{j}+\cdots\right] \\
& -\frac{1}{2} \boldsymbol{\nabla} \cdot \sum_{i} g_{i} \sum_{j}\left(\boldsymbol{y}_{i j}^{\mathrm{m}}-\boldsymbol{y}_{i}\right)\left(\boldsymbol{y}_{i j}^{\mathrm{m}}-\boldsymbol{y}_{i}\right) \\
& \times\left[\boldsymbol{R}_{i j}^{\mathrm{FU}} \cdot\left(\boldsymbol{u}_{j}-\langle\boldsymbol{u}\rangle_{j}\right)+\boldsymbol{R}_{i j}^{\mathrm{F} \Omega} \cdot\left(\boldsymbol{\omega}_{j}-\langle\boldsymbol{\omega}\rangle_{j}\right)\right. \\
& \left.-\boldsymbol{R}_{i j}^{\mathrm{FE}}:\langle\boldsymbol{e}\rangle_{j}+\cdots\right]+\cdots .
\end{aligned}
$$

The expansions for $\left\langle f^{\mathrm{h}}\right\rangle^{\mathrm{p}}$ and $\left\langle\boldsymbol{\sigma}^{\mathrm{h}}\right\rangle^{\mathrm{p}}$ may be continued to any order in $a / L$ and $\ell / L$ by retaining sufficient number of terms in Eq. (57) and the Taylor expansion for $g_{i j}^{\mathrm{m}}$ [see Eq. (51)]. The leading terms that are not given explicitly in Eqs. (65) and (66) are smaller by $\mathrm{O}(a / L)$ and $\mathrm{O}(\ell / L)$, respectively, than the smallest terms that are explicitly quoted. At high particle concentrations, the particles are near contact, and lubrication forces dominate. In such situations, $\boldsymbol{y}_{i j}^{\mathrm{m}} \approx \boldsymbol{y}_{j i}^{\mathrm{c}}$, and the particle phase stress resembles the contact stress. However, there exists a volume-averaged interphase drag, of which there is no equivalent for contact forces.

As a consequence of Eqs. (52) and (62), the momentum balance for the particle phase in a Stokesian suspension Eq. (46) assumes the form 


$$
0=n v^{\mathrm{p}}\left\langle\boldsymbol{b}^{\mathrm{ext}}\right\rangle^{\mathrm{p}}+n\left\langle\boldsymbol{f}^{\mathrm{h}}\right\rangle_{\mathrm{drag}}+\boldsymbol{\nabla} \cdot\left(n\langle\boldsymbol{\sigma}\rangle^{\mathrm{p}}\right),
$$

where $\langle\boldsymbol{\sigma}\rangle^{\mathrm{p}} \equiv\left\langle\boldsymbol{\sigma}^{\mathrm{h}}\right\rangle^{\mathrm{p}}+\left\langle\boldsymbol{\sigma}^{\mathrm{c}}\right\rangle^{\mathrm{p}}+\left\langle\boldsymbol{\sigma}^{\mathrm{ip}}\right\rangle^{\mathrm{p}}$. The interphase drag $\left\langle\boldsymbol{f}^{\mathrm{h}}\right\rangle_{\text {drag }}$ is given by Eq. (65), the hydrodynamic stress $\left\langle\boldsymbol{\sigma}^{\mathrm{h}}\right\rangle^{\mathrm{p}}$ by Eq. (64), the contact stress $\left\langle\boldsymbol{\sigma}^{\mathrm{c}}\right\rangle^{\mathrm{p}}$ by Eq. (53), and the stress due to interparticle forces $\left\langle\boldsymbol{\sigma}^{\text {ip }}\right\rangle^{p}$ by Eq. (55). Thus, we see that a well-defined particle phase stress exists. While the particle phase stress has hydrodynamic, contact, and interparticle force contributions, the interphase drag is purely hydrodynamic.

\section{DISCUSSION}

Comparison of the momentum balances for the particle phase Eq. (67) and the entire suspension Eq. (40) makes it clear that the surface traction due to particle contact makes identical contributions to the suspension and particle phase stresses, but the contributions of the fluid traction to the two stresses are quite different. The hydrodynamic part of $\langle\boldsymbol{\sigma}\rangle^{\mathrm{s}}$ arises from moments of the fluid traction about the particle center, while the hydrodynamic part of $\langle\boldsymbol{\sigma}\rangle^{\mathrm{p}}$ arises from moments of the hydrodynamic interaction forces about the particle center (with the interaction forces treated as acting at the midpoint of the separation between the particles). Thus, the micromechanical origin of the two contributions are distinct. At the lowest order in $(a / L)$, the hydrodynamic contribution to $\langle\boldsymbol{\sigma}\rangle^{\mathrm{s}}$ is $n\left\langle\overline{\boldsymbol{S}}^{\mathrm{h}}\right\rangle^{\mathrm{p}}$, as shown by Batchelor, ${ }^{3}$ while the hydrodynamic contribution to $\langle\boldsymbol{\sigma}\rangle^{\mathrm{p}}$ is $\sum_{i, j} g_{i}\left(\boldsymbol{y}_{i j}^{\mathrm{m}}-\boldsymbol{y}_{i}\right) \boldsymbol{f}_{i j}^{\mathrm{h}}$.

From Eqs. (63) and (64), it is clear that $\left\langle\boldsymbol{\sigma}^{\mathrm{h}}\right\rangle^{\mathrm{p}}$ arises solely from the hydrodynamic interactions between particles, but $\left\langle f^{\mathrm{h}}\right\rangle_{\text {drag }}$ can exist even in the absence of interactions. When hydrodynamic interactions are present, the relation for $\left\langle f^{\mathrm{h}}\right\rangle_{\text {drag }}$ is more complex than what intuition suggests: it is not simply the average of the hydrodynamic forces on the particles due to their own motion relative to the suspension, but also involves the hydrodynamic interaction forces between the particles. We emphasize that the relations for $\mathbf{\Sigma}^{(\mathrm{p})}$, $\langle\boldsymbol{\sigma}\rangle^{\mathrm{p}}$, and $\left\langle\boldsymbol{f}^{\mathrm{h}}\right\rangle_{\text {drag }}$ given in this paper are averages over particles and pairs of particles of micromechanical quantities. To achieve closure of the continuum equations, the averages of the micromechanical quantities must yield relations which are posed in terms of macroscopic averages of the kinematic variables. This can be accomplished by theoretical analysis for dilute suspensions, and by Stokesian Dynamics simulation $^{30,31}$ for arbitrary concentrations. Our relations for $\left\langle\boldsymbol{f}^{\mathrm{h}}\right\rangle_{\text {drag }}$ and $\langle\boldsymbol{\sigma}\rangle^{\mathrm{p}}$ will help in evaluating phenomenological models, such as the one advanced by Lhuillier. ${ }^{10}$

A notable point is that though the expressions for the interphase drag and hydrodynamic part of the particle phase stress in Eqs. (65) and (66) hold only for suspensions of rigid particles in Stokes flow, the validity of the expressions in Eqs. (63) and (64) is more general. This has the important proviso that a unique partition of the net hydrodynamic force on each particle into pairwise components, as in Eq. (58), is possible. The linearity of Stokes equations results in a unique partitioning; perhaps it is also possible in other situations.

In the absence of nonhydrodynamic interparticle forces, it is clearly of interest to determine if particle migration is indeed caused by the particle phase stress, as proposed by the
SBM. If we suppose, as indicated by experiment and simulations on viscometric flows, that the driving force for migration is proportional to the gradient of the strain rate, $\nabla\langle\boldsymbol{e}\rangle$, it is not immediately clear from the forms of $\left\langle f^{\mathrm{h}}\right\rangle_{\text {drag }}$ and $\boldsymbol{\nabla} \cdot\left(n\left\langle\boldsymbol{\sigma}^{\mathrm{h}}\right\rangle^{\mathrm{p}}\right)$ as to which drives migration-the expressions for both can have terms proportional to $\boldsymbol{\nabla}\langle\boldsymbol{e}\rangle$ if they are continued to sufficiently high order in $a / L$ and $\ell / L$. However, such a term occurs at $\mathrm{O}\left(a^{2} / L^{2}\right)$ in $\left\langle f^{\mathrm{h}}\right\rangle_{\text {drag }}$, and at $\mathrm{O}\left(a \ell / L^{2}\right)$ in $\boldsymbol{\nabla} \cdot\left(n\left\langle\boldsymbol{\sigma}^{\mathrm{h}}\right\rangle^{\mathrm{p}}\right)$, when compared to the leading term in $\left\langle f^{\mathrm{h}}\right\rangle$. Hence, if the separation of scales [Eq. (9)] holds, the latter is of greater magnitude, and will therefore be primarily responsible for particle migration. The above is not a rigorous argument, because it ignores factors such as the development of an anisotropic microstructure, which may impact the two terms differently. A proper investigation of the forms of $\left\langle f^{\mathrm{h}}\right\rangle_{\mathrm{drag}}$ and $\langle\boldsymbol{\sigma}\rangle^{\mathrm{p}}$ is necessary, but we may state with confidence that a term of the form $f_{d}(\phi) \eta / a^{2}\left(\langle\boldsymbol{u}\rangle^{\mathrm{p}}-\langle\boldsymbol{u}\rangle\right)$, which is conventionally given for the interphase drag, is part of $n\left\langle f^{\mathrm{h}}\right\rangle_{\text {drag }}$.

It is useful to relax our definition of Stokesian suspensions by removing the restriction that the Péclet number be large, and ask how Brownian motion affects the stresses of the solid/particle and fluid phases. The Brownian contribution to the stress in a sheared suspension has been considered by several authors, but the study whose results can be directly used by us is that of Brady. ${ }^{17}$ He showed that there are two direct Brownian contributions to the suspension stress, namely the isotropic kinetic contribution $-n k T I$, and the contribution $n\left\langle\boldsymbol{S}^{\mathrm{B}}\right\rangle$ due to the stresslets that are induced on the particles in response to their Brownian motion. Brady showed that $n\left\langle\boldsymbol{S}^{\mathrm{B}}\right\rangle=-n k T\left\langle\boldsymbol{\nabla}_{\mathrm{c}} \cdot\left[\boldsymbol{R}^{\mathrm{SU}} \cdot\left(\boldsymbol{R}^{\mathrm{FU}}\right)^{-1}\right]\right\rangle$ where $\boldsymbol{\nabla}_{\mathrm{c}}$ is the configurational gradient, and is contracted with the last index of $\left(\boldsymbol{R}^{\mathrm{FU}}\right)^{-1}$. But regardless of the relation for $n\left\langle\boldsymbol{S}^{\mathrm{B}}\right\rangle$, we have shown that the stresslet contributes only to the fluid phase stress, not to the solid (or particle) phase stress; hence $n\left\langle S^{\mathrm{B}}\right\rangle$ is the direct Brownian contribution to the fluid phase stress. The isotropic part $-n k T I$ comes from the thermal motion of the particles, and is therefore a part of the particle phase stress. Some previous studies ${ }^{33,34}$ that considered the influence of Brownian motion on particle migration have added the entire direct Brownian stress to the particle phase stress; from the arguments advanced above, this appears to be incorrect.

\section{CONCLUSIONS}

The central thesis of the suspension balance model, proposed by Nott and Brady, ${ }^{2}$ is that particle migration in Stokesian suspensions is driven by the divergence of the particle phase stress $\langle\boldsymbol{\sigma}\rangle^{\mathrm{p}}$. They equated this stress to the "particle stress" $\Sigma^{(p)}$, defined by Batchelor ${ }^{3}$ as the particle contribution to the suspension stress. On the other hand, the equations of motion for two-phase flows developed in numerous studies $^{4,7,9}$ show clearly that the volume-averaged (or ensemble-averaged) momentum balance for the particle phase has only a force $\langle f\rangle^{\mathrm{p}}$-the "particle stress" $\boldsymbol{\Sigma}^{(\mathrm{p})}$ plays no role in this equation. This discrepancy went unnoticed, until it was pointed out recently by Lhuillier. ${ }^{10}$ 
In this paper we reaffirm the observation of Lhuillier. ${ }^{10}$ However, we have shown that the hydrodynamic part of the particle phase force $\left\langle f^{\mathrm{h}}\right\rangle^{\mathrm{p}}$ can, and indeed should, be written as the sum of an interphase drag $\left\langle f^{\mathrm{h}}\right\rangle_{\text {drag }}$ and the divergence of a hydrodynamic stress $n\left\langle\boldsymbol{\sigma}^{\mathrm{h}}\right\rangle^{\mathrm{p}}$. This partitioning of $\left\langle f^{\mathrm{h}}\right\rangle^{\mathrm{p}}$ is not arbitrary, but arises naturally; the method used is the exact analog of the derivation of the stress due to any pairwise additive nonhydrodynamic force. Thus, though the suspension balance model errs in equating the particle phase stress to the particle contribution to the suspension stress, its form is correct. By a rigorous volume averaging procedure, we have determined exact micromechanical relations for the drag $\left\langle f^{\mathrm{h}}\right\rangle_{\text {drag }}$ and the hydrodynamic particle phase stress $\left\langle\boldsymbol{\sigma}^{\mathrm{h}}\right\rangle^{\mathrm{p}}$. This stress differs fundamentally from the particle contribution to the hydrodynamic part of the suspension stress $\Sigma^{\mathrm{h}(\mathrm{p})}$ : the latter is related to the moments of the fluid traction on the surface of the particles about their centers (such as the stresslet), while the former is related to the moments of the hydrodynamic interaction forces between particles about their centers, with the interaction forces treated as acting at the midpoint of the separation between the particles. The drag force too is more complicated than what intuition suggests: it is not simply the average force on the particles due to their motion relative to the suspension, but also involves the hydrodynamic interaction forces. We show by an order of magnitude estimate that particle migration is likely to be driven by the divergence of the particle phase stress $\left\langle\boldsymbol{\sigma}^{\mathrm{h}}\right\rangle^{\mathrm{p}}$; however a thorough investigation of the interphase force $\left\langle\boldsymbol{f}^{\mathrm{h}}\right\rangle_{\text {drag }}$ and the particle phase stress $\left\langle\boldsymbol{\sigma}^{\mathrm{h}}\right\rangle^{\mathrm{p}}$, either by analytical calculation or by Stokesian Dynamics simulation, is necessary to make a definitive statement.

Despite the aforementioned flaws, many studies ${ }^{19-21,24}$ have used the suspension balance model with success to capture the observed features of particle migration in many simple flows. This suggests that the phenomenological form of $\boldsymbol{\Sigma}^{\mathrm{h}(\mathrm{p})}$ assumed in these studies may be qualitatively similar to that of the particle phase stress. In this paper, we have given micromechanical relations for $\left\langle\boldsymbol{f}^{\mathrm{h}}\right\rangle_{\text {drag }}$ and $\left\langle\boldsymbol{\sigma}^{\mathrm{h}}\right\rangle^{\mathrm{p}}$-it remains to achieve closure by deriving relations for them in terms of volume-averaged kinematic quantities, such as the average velocities and of the suspension and the particle phase and their frame-invariant gradients.

Particle migration could also be driven by the divergence of the particle phase contact stress $\left\langle\boldsymbol{\sigma}^{\mathrm{c}}\right\rangle^{\mathrm{p}}$ (called the IrvingKirwood stress by Lhuillier $\left.{ }^{10}\right)$. Unlike the hydrodynamic part of the particle phase stress $\left\langle\boldsymbol{\sigma}^{\mathrm{h}}\right\rangle^{\mathrm{p}}$, the contact stress contributes also to the suspension stress $\langle\boldsymbol{\sigma}\rangle$. We expect $\left\langle\boldsymbol{\sigma}^{\mathrm{c}}\right\rangle^{\mathrm{p}}$ to increase rapidly with increasing $\phi$; hence it may well be one of the causes of particle migration in concentrated suspensions.

Our paper has some implications for the interpretation of rheological data on Stokesian suspensions. Many previous studies have determined the particle phase pressure by equating it to the isotropic part of the $\Sigma^{(p)}{ }^{12-15}$ - in light of our results, this is incorrect. Indeed, the particle phase pressure, or for that matter any component of the particle phase stress tensor, cannot be determined directly from rheological measurements of the suspension.

\section{ACKNOWLEDGMENTS}

This paper is the product of a collaboration supported by the Indo-French Center for the Promotion of Advanced Research. We thank John F. Brady, Bud Homsy, Daniel Lhuillier, Jeffrey Morris, and Ganesh Subramanian for extensive discussions.

\section{APPENDIX A: VOLUME-AVERAGED MOMENTUM BALANCES INCLUDING INERTIAL CONTRIBUTIONS}

Here we derive the complete volume-averaged momentum balances, including the effects of particle and fluid inertia. The suspension momentum balance is obtained by multiplying Eq. (17) by $g$ and integrating over the entire volume,

$$
\int g \rho \frac{\hat{\mathrm{D}} \boldsymbol{u}}{\hat{\mathrm{D}} t} \mathrm{~d} V=\int g \hat{\boldsymbol{\nabla}} \cdot \boldsymbol{\sigma} \mathrm{d} V+\int g \boldsymbol{b} \mathrm{d} V .
$$

The left hand side may be simplified using Eq. (16), to yield

$$
\frac{\partial}{\partial t} \int g \boldsymbol{\mu} \boldsymbol{d} V+\int \hat{\boldsymbol{\nabla}} \cdot(g \rho \boldsymbol{u} \boldsymbol{u}) \mathrm{d} V-\int \boldsymbol{\rho} \boldsymbol{u} \cdot \hat{\boldsymbol{\nabla}} g \mathrm{~d} V .
$$

The second term vanishes on application of the divergence theorem and using Eq. (7). Using Eq. (6), Eq. (A2) reduces to

$$
\frac{\partial}{\partial t}\langle\rho \boldsymbol{u}\rangle+\nabla \cdot\langle\rho \boldsymbol{u} \boldsymbol{u}\rangle
$$

The integration of the right-hand side proceeds in the same manner as in §II; combining Eq. (A3) with the right-hand side of Eq. (29), the volume-averaged suspension momentum balance with allowance for inertia is

$$
\frac{\partial}{\partial t}\langle\rho \boldsymbol{u}\rangle+\boldsymbol{\nabla} \cdot\langle\rho \boldsymbol{u} \boldsymbol{u}\rangle=\boldsymbol{\nabla} \cdot\left[(1-\phi)\langle\boldsymbol{\sigma}\rangle^{\mathrm{f}}+\phi\langle\boldsymbol{\sigma}\rangle^{\mathrm{s}}\right]+\langle\boldsymbol{b}\rangle .
$$

The expressions for the fluid and solid phase stresses are given in Eqs. (30) and (32), respectively, which are

$$
(1-\phi)\langle\boldsymbol{\sigma}\rangle^{\mathrm{f}}=-(1-\phi)\langle p\rangle^{\mathrm{f}} \boldsymbol{I}+2 \eta\langle\boldsymbol{e}\rangle,
$$

and

$$
\phi\langle\boldsymbol{\sigma}\rangle^{\mathrm{s}}=\sum_{i} g_{i} \int_{V_{i}} \boldsymbol{\sigma} \mathrm{d} V-\nabla \cdot \sum_{i} g_{i} \int_{V_{i}} \boldsymbol{y}^{\prime} \boldsymbol{\sigma} \mathrm{d} V+\cdots .
$$

As in §II, we transform every term in Eq. (A6) using Eqs. (33) and (B5) to bring them to a more useful form. Inertia has no direct effect on the surface integrals in the transformed relation, but alters the volume integrals when we substitute $\hat{\boldsymbol{\nabla}} \cdot \boldsymbol{\sigma}=\rho \hat{\mathrm{D}} \boldsymbol{u} / \hat{\mathrm{D}} t-\boldsymbol{b}$. The pointwise velocity in a rigid particle is $\boldsymbol{u}=\boldsymbol{u}_{i}+\boldsymbol{\omega}_{i} \times \boldsymbol{r}$, where $\boldsymbol{u}_{i}$ and $\boldsymbol{\omega}_{i}$ are the translational and angular velocities, respectively, of its center. As a result, $\hat{\mathrm{D}} \boldsymbol{u} / \hat{\mathrm{D}} t=\dot{\boldsymbol{u}}_{i}+\dot{\boldsymbol{\omega}}_{i} \times \boldsymbol{y}^{\prime}+\boldsymbol{\omega}_{i} \boldsymbol{\omega}_{i} \cdot \boldsymbol{y}^{\prime}-\boldsymbol{\omega}_{i} \cdot \boldsymbol{\omega}_{i} \boldsymbol{y}^{\prime}$, and hence the first term in Eq. (A6) takes the form 


$$
\begin{aligned}
\sum_{i} g_{i} \int_{V_{i}} \boldsymbol{\sigma} \mathrm{d} V= & \sum_{i} g_{i} \int_{S_{i}} \boldsymbol{y}^{\prime} \boldsymbol{n} \cdot \boldsymbol{\sigma} \mathrm{d} S \\
& -\sum_{i} g_{i} \int_{V_{i}} \boldsymbol{y}^{\prime}\left[\left(\rho \dot{\boldsymbol{u}}_{i}-\boldsymbol{b}\right)\right. \\
& \left.+\rho\left(\dot{\boldsymbol{\omega}}_{i} \times \boldsymbol{y}^{\prime}+\boldsymbol{\omega}_{i} \boldsymbol{\omega}_{i} \cdot \boldsymbol{y}^{\prime}-\boldsymbol{\omega}_{i} \cdot \boldsymbol{\omega}_{i} \boldsymbol{y}^{\prime}\right)\right] \mathrm{d} V .
\end{aligned}
$$

Substituting Eq. (A7) and the similarly simplified higherorder terms in Eq. (A6), we get

$$
\begin{aligned}
\phi\langle\boldsymbol{\sigma}\rangle^{\mathrm{S}}= & \sum_{i} g_{i} \boldsymbol{S}_{i}-\frac{1}{2} \boldsymbol{\nabla} \cdot \sum_{i} g_{i} \boldsymbol{Q}_{i}+\cdots \\
& +\sum_{i} g_{i}\left(\int_{V_{i}} \boldsymbol{y}^{\prime}\left(\rho \dot{\boldsymbol{u}}_{i}-\boldsymbol{b}\right) \mathrm{d} V+\frac{\mathcal{I}}{2}\left(-\boldsymbol{\epsilon} \cdot \dot{\boldsymbol{\omega}}_{i}+\boldsymbol{\omega}_{i} \cdot \boldsymbol{\omega}_{i} \boldsymbol{I}\right.\right. \\
& \left.\left.-\boldsymbol{\omega}_{i} \boldsymbol{\omega}_{i}\right)\right)+\frac{1}{2} \boldsymbol{\nabla} \cdot\left(\sum_{i} g_{i} \int_{V_{i}} \boldsymbol{y}^{\prime} \boldsymbol{y}^{\prime}\left(\rho \dot{\boldsymbol{u}}_{i}-\boldsymbol{b}\right) \mathrm{d} V\right) \\
& +\cdots
\end{aligned}
$$

where $\mathcal{I} \equiv\left(\frac{2}{5}\right) \rho^{\mathrm{p}} v^{\mathrm{p}} a^{2}$ is the moment of inertia of the spherical particles, and we have followed the notation for the surface moments defined in Eq. (36). In Eq. (A8), the terms in the first line arise from the moments of the surface traction, namely the first term on the right-hand-side of Eq. (34) and the first term in Eq. (B5); the subsequent terms arise from the volume moments of the inertia and body forces, namely the second term on the right-hand-side of Eq. (34) and the second term in Eq. (B5). Decomposing the body force density in particle $i$ as the sum of its mean $\boldsymbol{b}_{i}$ and a deviation $\boldsymbol{b}^{\prime}$, and using of the equations of linear and angular momentum for the particle,

$$
m \dot{\boldsymbol{u}}_{i}=\boldsymbol{f}_{i}+\boldsymbol{b}_{i} v^{\mathrm{p}}, \quad \mathcal{I} \dot{\boldsymbol{\omega}}_{i}=-\boldsymbol{\epsilon}: \int_{S_{i}} \boldsymbol{y}^{\prime}(\boldsymbol{n} \cdot \boldsymbol{\sigma}) \mathrm{d} S+\boldsymbol{\tau}_{i},
$$

where $\boldsymbol{\tau}_{i}$ is the net external torque on particle $i$, Eq. (A8) may be simplified to

$$
\begin{aligned}
\phi\langle\boldsymbol{\sigma}\rangle^{\mathrm{s}}= & \sum_{i} g_{i}\left(\overline{\boldsymbol{S}}_{i}-\frac{1}{2} \boldsymbol{\epsilon} \cdot \boldsymbol{\tau}_{i}+\frac{1}{2} \mathcal{I}\left(\boldsymbol{\omega}_{i} \cdot \boldsymbol{\omega}_{i} \boldsymbol{I}-\boldsymbol{\omega}_{i} \boldsymbol{\omega}_{i}\right)\right. \\
& \left.+\int_{V_{i}} \boldsymbol{y}^{\prime} \boldsymbol{b}^{\prime} \mathrm{d} V\right)-\frac{1}{2} \boldsymbol{\nabla} \cdot \sum_{i} g_{i}\left(\boldsymbol{Q}_{i}-\frac{1}{5} v^{\mathrm{p}} a^{2} \boldsymbol{I} \boldsymbol{f}_{i}\right. \\
& \left.+\int_{V_{i}} \boldsymbol{y}^{\prime} \boldsymbol{y}^{\prime} \boldsymbol{b}^{\prime} \mathrm{d} V\right)+\cdots
\end{aligned}
$$

In the derivation of Eqs. (A8) and (A10), we have used the identities $\int_{V_{i}} \boldsymbol{y}^{\prime} \mathrm{d} V=0$ and $\int_{V_{i}} \boldsymbol{y}^{\prime} \boldsymbol{y}^{\prime} \rho \mathrm{d} V=\left(\frac{1}{5}\right) \rho^{\mathrm{p}} v^{\mathrm{p}} a^{2} \boldsymbol{I}$.

Substituting Eqs. (A5) and (A10) in Eq. (A4), the momentum balance for the suspension takes the form

$$
\begin{aligned}
\frac{\partial}{\partial t}\left(\langle\rho\rangle\langle\boldsymbol{u}\rangle_{\mathrm{m}}\right)+\nabla \cdot\left(\langle\rho\rangle\langle\boldsymbol{u}\rangle_{\mathrm{m}}\langle\boldsymbol{u}\rangle_{\mathrm{m}}\right) \\
=\langle\boldsymbol{b}\rangle-\nabla\left[(1-\phi)\langle p\rangle^{\mathrm{f}}\right]+2 \eta \nabla \cdot\langle\boldsymbol{e}\rangle+\boldsymbol{\nabla} \cdot\left[\boldsymbol{\phi}\langle\boldsymbol{\sigma}\rangle^{\mathrm{s}}\right. \\
\left.\quad+\left\langle\rho \boldsymbol{u}^{\prime} \boldsymbol{u}^{\prime}\right\rangle\right] .
\end{aligned}
$$

where $\langle\boldsymbol{u}\rangle_{\mathrm{m}}$ is the mass-averaged suspension velocity, defined in Eq. (25), and $\boldsymbol{u}^{\prime} \equiv \boldsymbol{u}-\langle\boldsymbol{u}\rangle_{\mathrm{m}}$ is the deviation of the local velocity from $\langle\boldsymbol{u}\rangle_{\mathrm{m}}$. The sum of $\phi\langle\boldsymbol{\sigma}\rangle^{\mathrm{s}}$ and the Reynolds's stress $\left\langle\rho \boldsymbol{u}^{\prime} \boldsymbol{u}^{\prime}\right\rangle$ is the "particle stress" $\boldsymbol{\Sigma}^{(\mathrm{p})}$, introduced by Batchelor $^{3}$ as the particle contribution to the suspension stress.

Next, we determine the momentum balance for the solid phase by multiplying Eq. (17) by $\chi g$ and integrating over the entire volume. Using Eqs. (3), (4), and (16), and employing the same manipulations as in Eq. (A2), we get

$$
\begin{aligned}
& \rho^{\mathrm{p}} \frac{\partial}{\partial t}\left(\phi\langle\boldsymbol{u}\rangle^{\mathrm{s}}\right)+\rho^{\mathrm{p}} \boldsymbol{\nabla} \cdot\left(\phi\langle\boldsymbol{u} \boldsymbol{u}\rangle^{\mathrm{s}}\right) \\
& \quad=\boldsymbol{\nabla} \cdot\left(\phi\langle\boldsymbol{\sigma}\rangle^{\mathrm{s}}\right)+\sum_{i} \int_{S_{i}} \boldsymbol{n} \cdot \boldsymbol{\sigma} g \mathrm{~d} S+\phi\langle\boldsymbol{b}\rangle^{\mathrm{s}} .
\end{aligned}
$$

To evaluate the integral in Eq. (A12), we substitute the Taylor expansion of $g$ from Eq. (31); the result is

$$
\begin{aligned}
\sum_{i} \int_{S_{i}} \boldsymbol{n} \cdot \boldsymbol{\sigma} g \mathrm{~d} S= & \sum_{i} g_{i} \boldsymbol{f}_{i}-\boldsymbol{\nabla} \cdot \sum_{i} g_{i} \boldsymbol{S}_{i} \\
& +\frac{1}{2} \boldsymbol{\nabla} \nabla: \sum_{i} g_{i} \boldsymbol{Q}_{i} \cdots
\end{aligned}
$$

On substituting in Eq. (A12) the expansions for $\boldsymbol{\nabla} \cdot\left(\phi\langle\boldsymbol{\sigma}\rangle^{\mathrm{s}}\right)$ from Eq. (A8) and $\sum_{i} \int_{S_{i}} \boldsymbol{n} \cdot \boldsymbol{\sigma} g \mathrm{~d} S$ from Eq. (A13), it is immediately clear that all the terms in the latter, except the first, are cancelled by the terms in the first line of the former. With the cancellations, the solid phase momentum balance reduces to

$$
\begin{aligned}
\rho^{\mathrm{p}} \frac{\partial}{\partial t}\left(\phi\langle\boldsymbol{u}\rangle^{\mathrm{s}}\right)+\rho^{\mathrm{p}} \boldsymbol{\nabla} \cdot\left(\boldsymbol{\phi}\langle\boldsymbol{u} \boldsymbol{u}\rangle^{\mathrm{s}}\right) & \\
= & \phi\langle\boldsymbol{b}\rangle^{\mathrm{s}}+\sum_{i} g_{i} \boldsymbol{f}_{i}+\boldsymbol{\nabla} \cdot\left\{\sum _ { i } g _ { i } \left[\int_{V_{i}} \boldsymbol{y}^{\prime} \boldsymbol{b}^{\prime} \mathrm{d} V\right.\right. \\
& \left.+\frac{\mathcal{I}}{2}\left(-\boldsymbol{\epsilon} \cdot \dot{\boldsymbol{\omega}}_{i}+\boldsymbol{\omega}_{i} \cdot \boldsymbol{\omega}_{i} \boldsymbol{I}-\boldsymbol{\omega}_{i} \boldsymbol{\omega}_{i}\right)\right] \\
& \left.+\frac{1}{2} \boldsymbol{\nabla} \cdot \sum_{i} g_{i} \int_{V_{i}} \boldsymbol{y}^{\prime} \boldsymbol{y}^{\prime}\left(\rho \dot{\boldsymbol{u}}_{i}-\boldsymbol{b}\right) \mathrm{d} V+\cdots\right\} .
\end{aligned}
$$

Though Eq. (A14) is adequate, it can be simplified considerably in the following way. As noted earlier in this section, the terms within the square brackets are volume moments about the particle centers of $\rho D \boldsymbol{u} / D t-\boldsymbol{b}$, starting from the first moment. The left hand side of Eq. (A14) and the body force $\phi\langle\boldsymbol{b}\rangle^{\mathrm{s}}$ may also be written as the sum of moments, by replacing $g$ with its Taylor expansion Eq. (31). We then find that all moments, except the zeroth, of the left hand side and the body force cancel exactly with the terms within the square brackets of Eq. (A14), yielding 


$$
\begin{gathered}
\rho^{\mathrm{p}} v^{\mathrm{p}} \frac{\partial}{\partial t}\left(\sum_{i} g_{i} \boldsymbol{u}_{i}\right)+\rho^{\mathrm{p}} v^{\mathrm{p}} \nabla \cdot\left(\sum_{i} g_{i} \boldsymbol{u}_{i} \boldsymbol{u}_{i}\right) \\
=v^{\mathrm{p}} \sum_{i} g_{i} \boldsymbol{b}_{i}+\sum_{i} g_{i} \boldsymbol{f}_{i} .
\end{gathered}
$$

Decomposing $v^{\mathrm{p}} \boldsymbol{b}_{i}$ into an external body force $v^{\mathrm{p}} \boldsymbol{b}_{i}^{\text {ext }}$ and an 'action at a distance' interparticle force $f_{i}^{\text {ip }}$, and recognizing that every term in this equation is a particle phase average [defined in Eq. (15)], we get

$$
\rho^{\mathrm{p}} v^{\mathrm{p}}\left[\frac{\partial}{\partial t}\left(n\langle\boldsymbol{u}\rangle^{\mathrm{p}}\right)+\boldsymbol{\nabla} \cdot\left(n\langle\boldsymbol{u} \boldsymbol{u}\rangle^{\mathrm{p}}\right)\right]=n v^{\mathrm{p}}\left\langle\boldsymbol{b}^{\text {ext }}\right\rangle^{\mathrm{p}}+n\langle\boldsymbol{f}\rangle^{\mathrm{p}} .
$$

This is clearly the momentum balance for the particle phase, as the left hand side is the average rate of change of momentum of the particle phase. As we have shown above, it is simply another form of the momentum balance for the solid phase Eq. (A14).

\section{APPENDIX B: TRANSFORMATION OF VOLUME INTEGRALS OF THE STRESS MOMENTS}

As we are interested in the divergence of $\phi\langle\boldsymbol{\sigma}\rangle^{\mathrm{s}}$ and not the stress itself, we consider the divergence of the $n^{\text {th }}$ term $(n>1)$ in the right-hand side of Eq. (32). The manipulations are greatly eased by adopting the index notation, with the Einstein convention of summing over repeated indices. The $n^{\text {th }}$ term then is

$$
\frac{(-1)^{(n-1)}}{(n-1) !} \partial_{p_{1}} \partial_{p_{2}} \cdots \partial_{p_{n-1}} \partial_{p_{n}} \sum_{i} g_{i} \int_{V_{i}} y_{p_{1}}^{\prime} y_{p_{2}}^{\prime} \cdots y_{p_{n-1}}^{\prime} \sigma_{p_{n} q} \mathrm{~d} V,
$$

where we have used the notation $\partial_{k} \equiv \partial / \partial x_{k}$. To bring this to the desired form, we rewrite the integrand as

$$
\begin{aligned}
& \prod_{r=1}^{n-1} y_{p_{r}}^{\prime} \sigma_{p_{n} q}=\frac{1}{n}\left[\sum_{m=1}^{n} \sigma_{p_{m} q} \prod_{\substack{r=1 \\
r \neq m}}^{n} y_{p_{r}}^{\prime}\right. \\
& \left.+\sum_{m=1}^{n-1}\left(y_{p_{m}}^{\prime} \sigma_{p_{n} q}-y_{p_{n}}^{\prime} \sigma_{p_{m} q}\right) \prod_{\substack{r=1 \\
r \neq m}}^{n-1} y_{p_{r}}^{\prime}\right] \text {. }
\end{aligned}
$$

Notice that each term in the second sum on the right-hand side of Eq. (B2)) is antisymmetric in the indices $p_{m}, p_{n}$. Hence the integral too is antisymmetric in these indices, and will vanish upon the operation $\partial_{p_{m}} \partial_{p_{n}}$. As a result, only the terms in the first sum of Eq. (B2) are of consequence; this sum may be written as

$$
\sum_{m=1}^{n} \sigma_{p_{m} q} \prod_{\substack{r=1 \\ r \neq m}}^{n} y_{p_{r}}^{\prime}=\partial_{m}\left(\prod_{r=1}^{n} y_{p_{r}}^{\prime} \sigma_{m q}\right)-\prod_{r=1}^{n} y_{p_{r}}^{\prime} \partial_{m} \sigma_{m q}
$$

The expression in Eq. (B1) then takes the form

$$
\begin{aligned}
& -\frac{(-1)^{n}}{n !} \partial_{p_{1}} \partial_{p_{2}} \cdots \partial_{p_{n-1}} \partial_{p_{n}} \sum_{i} g_{i} \int_{V_{i}} \\
& \quad \times\left[\partial_{m}\left(\prod_{r=1}^{n} y_{p_{r}}^{\prime} \sigma_{m q}\right)-\prod_{r=1}^{n} y_{p_{r}}^{\prime} \partial_{m} \sigma_{m q}\right] \mathrm{d} V
\end{aligned}
$$

The integral of the first term in the square brackets in Eq. (B4) reduces to a surface integral over $S_{i}$, by the divergence theorem. For the second term, we substitute for $\partial_{k} \sigma_{k q}$ from Eq. (17), and write the integral as a volume moment of the force on the particle, including inertia. The above expression thus reduces to

$$
\begin{gathered}
-\frac{(-1)^{n}}{n !} \partial_{p_{1}} \cdots \partial_{p_{n}} \sum_{i} g_{i}\left[\int_{S_{i}} y_{p_{1}}^{\prime} \cdots y_{p_{n}}^{\prime} n_{k} \sigma_{k q} \mathrm{~d} S\right. \\
\left.-\int_{V_{i}} y_{p_{1}}^{\prime} \cdots y_{p_{n}}^{\prime}\left(\rho \frac{\mathrm{D} u_{q}}{\mathrm{D} t}-b_{q}\right) \mathrm{d} V\right],
\end{gathered}
$$

which is the desired transformation of Eq. (B1). Prosperetti ${ }^{8}$ derived it for $n=3$, and stated that the result could be generalized for larger $n$. Our derivation is for arbitrary $n$.

${ }^{1}$ D. T. Leighton and A. Acrivos, "The shear-induced migration of particles in concentrated suspensions," J. Fluid Mech. 181, 415 (1987).

${ }^{2}$ P. R. Nott and J. F. Brady, "Pressure-driven flow of suspensions: Simulation and theory," J. Fluid Mech. 275, 157 (1994).

${ }^{3}$ G. K. Batchelor, "The stress system in a suspension of force-free particles," J. Fluid Mech. 41, 545 (1970).

${ }^{4}$ Y. A. Buyevich and I. N. Shchelchkova, "Flow of dense suspensions," Prog. Aerosp. Sci. 18, 121 (1978).

${ }^{5}$ D. A. Drew, "Mathematical modeling of two-phase flow," Annu. Rev. Fluid Mech. 15, 261 (1983).

${ }^{6} \mathrm{R}$. Jackson, "Locally averaged equations of motion for a mixture of identical particles and a Newtonian fluid," Chem. Eng. Sci. 52, 2457 (1997).

${ }^{7}$ D. Z. Zhang and A. Prosperetti, "Momentum and energy equations for disperse two-phase flows and their closure for dilute suspensions," Int. J. Multiphase Flow 23, 425 (1997).

${ }^{8}$ A. Prosperetti, "The average stress in incompressible disperse flow," Int. J. Multiphase Flow 30, 1011 (2004).

${ }^{9}$ T. B. Anderson and R. Jackson, "Fluid mechanical description of fluidized beds. equations of motion," Ind. Eng. Chem. Fundam. 6, 527 (1967).

${ }^{10} \mathrm{D}$. Lhuillier, "Migration of rigid particles in non-Brownian viscous suspensions," Phys. Fluids 21, 023302 (2009).

${ }^{11}$ D. J. Jeffrey, J. F. Morris, and J. F. Brady, "The pressure moments for two spheres in a low-Reynolds-number flow," Phys. Fluids A 5, 2317 (1993).

${ }^{12}$ J. F. Brady and J. F. Morris, "Microstructure of strongly sheared suspensions and its impact on rheology and diffusion," J. Fluid Mech. 348, 103 (1997).

${ }^{13}$ A. Singh and P. R. Nott, "Normal stresses and microstructure in bounded sheared suspensions via Stokesian dynamics simulations," J. Fluid Mech. 412, 279 (2000).

${ }^{14}$ I. E. Zarraga, D. A. Hill, and D. T. Leighton, "The characterization of the total stress of concentrated suspensions of noncolloidal spheres in Newtonian fluids," J. Rheol. 44, 185 (2000).

${ }^{15}$ A. Sierou and J. F. Brady, "Rheology and microstructure in concentrated non-colloidal suspensions," J. Rheol. 46, 1031 (2002).

${ }^{16}$ A. Singh and P. R. Nott, "Experimental measurements of the normal stresses in sheared Stokesian suspensions," J. Fluid Mech. 490, 293 (2003).

${ }^{17}$ J. F. Brady, "Brownian motion, hydrodynamics, and the osmotic pressure," J. Chem. Phys. 98, 3335 (1993).

${ }^{18}$ J. F. Morris and F. Boulay, "Curvilinear flows of noncolloidal suspensions: the role of normal stresses," J. Rheol. 43, 1213 (1999).

${ }^{19}$ R. M. Miller, J. P. Singh, and J. F. Morris, "Suspension flow modeling for general geometries," Chem. Eng. Sci. 64, 4597 (2009).

${ }^{20}$ J. F. Morris and J. F. Brady, "Pressure-driven flow of a suspension: Bbuoyancy effects," Int. J. Multiphase Flow 24, 105 (1998). 
${ }^{21}$ R. M. Miller and J. F. Morris, "Normal stress-driven migration and axial development in pressure-driven flow of concentrated suspensions," J. Non-Newtonian Fluid Mech. 135, 149 (2006).

${ }^{22}$ J. T. Norman, H. V. Nayak, and R. T. Bonnecaze, "Migration of buoyant particles in low-Reynolds-number pressure-driven flows," J. Fluid Mech. 523, 1 (2005).

${ }^{23}$ J. T. Norman, B. O. Oguntade, and R. T. Bonnecaze, "Particle-phase distributions of pressure-driven flows of bidisperse suspensions," J. Fluid Mech. 594, 1 (2008).

${ }^{24}$ K. Yapici, R. L. Powell, and R. J. Phillips, "Particle migration and suspension structure in steady and oscillatory plane Poiseuille flow," Phys. Fluids 21, 053302 (2009).

${ }^{25}$ A. Ramachandran and D. T. Leighton, "The effect of gravity on the meniscus accumulation phenomenon in a tube," J. Rheol. 51, 1073 (2007).

${ }^{26}$ A. Ramachandran and D. T. Leighton, "The influence of secondary flows induced by normal stress differences on the shear-induced migration of particles in concentrated suspensions," J. Fluid Mech. 603, 207 (2008).

${ }^{27}$ A. Ramachandran, M. Loewenberg, and D. T. Leighton, "A constitutive equation for droplet distribution in unidirectional flows of dilute emulsions for low capillary numbers," Phys. Fluids 22, 083301 (2010).

${ }^{28}$ S. Kim and W. B. Russel, "Modelling of porous media by renormalization of the stokes equations," J. Fluid Mech. 154, 269 (1985).

${ }^{29}$ G. K. Batchelor, "The effect of Brownian motion on the bulk stress in a suspension of spherical particles," J. Fluid Mech. 83, 97 (1977).

${ }^{30}$ J. F. Brady and G. Bossis, "Stokesian dynamics," Annu. Rev. Fluid Mech. 20, 111 (1988).

${ }^{31}$ J. F. Brady, R. J. Philips, J. C. Lester, and G. Bossis, "Dynamic simulation of hydrodynamically interacting suspensions," J. Fluid Mech. 195, 257 (1988).

${ }^{32}$ S. Kim and S. J. Karrila, Microhydrodynamics: Principles and Selected Applications (Dover, New York, 2005).

${ }^{33}$ M. Frank, D. Anderson, E. R. Weeks, and J. F. Morris, "Particle migration in pressure-driven flow of a Brownian suspension," J. Fluid Mech., 493, 363378 (2003)

${ }^{34}$ J. R. Brown, E. O. Fridjonsson, J. D. Seymour, and S. L. Codd, "Nuclear magnetic resonance measurement of shear-induced particle migration in Brownian suspensions," Phys. Fluids 21, 093301 (2009). 